\title{
Cooling out in der Arbeitswelt: Berufswechsel als Folge eines Mismatch von Habitus und Feld
}

\author{
Hildegard Matthies
}

Angenommen: 11. Oktober 2021 / Online publiziert: 4. November 2021

(C) Der/die Autor(en) 2021

Zusammenfassung In diesem Beitrag werden Berufswechsel aus der Cooling-outPerspektive rekonstruiert. Dabei wird das Cooling-out-Konzept in zweierlei Hinsicht erweitert. Erstens wird es mit Blick auf die an Goffman anschließende Diskussion nicht nur als eine von außen auf ein Subjekt gerichtete Selektionsstrategie aufgefasst, sondern auch als eine von innen kommende Strategie der Krisenintervention zur Verarbeitung von beruflichen Enttäuschungen. Zweitens wird ein berufliches Cooling out in Anlehnung an Bourdieu als Folge eines misslungenen Passungsverhältnisses von beruflichen Bewährungsbedingungen und individuellen Dispositionen konzeptualisiert, um die Entweder-oder-Struktur von Selbst- versus Fremdselektion zu überwinden und auch das ,Dazwischen“ von Feld und Subjekt in den Blick zu nehmen. Am Beispiel von empirischen Fallstudien unter Berufswechslern wird gezeigt, dass Entscheidungen für einen Berufswechsel keiner der beiden Seiten allein zugeschlagen werden können, sondern lediglich den Endpunkt einer kontinuierlichen biographischen Erfahrungsaufschichtung darstellen, an der individuelle Dispositionen ebenso wie Feldstrukturen ihren Anteil haben.

Schlüsselwörter Cooling out $\cdot$ Berufswechsel $\cdot$ Habitus $\cdot$ Berufliche Antriebsstruktur 


\title{
Cooling out in the working world: Career change as a consequence of mismatch between habitus and field
}

\begin{abstract}
In this paper, career changes are reconstructed from a cooling out perspective. To do so, Erving Goffman's concept of cooling out is amended in a twofold way: first, it is understood not only as a selection strategy confronting the subject from outside, but also as a strategy of crisis intervention coming from within to deal with professional disappointments. Second, following Bourdieu, a professional cooling out is conceptualized as the consequence of a mismatch between professional conditions of success and individual dispositions, the intention being to overcome the alternative of external vs. internal selection in favor of focusing on the space in between subject and field. Drawing upon empirical case studies among changers of occupation, it is shown that decisions for a career change can be attributed to neither side alone. Instead, they must be regarded as the end point of a continual biographical layering of experiences that is fed by both individual dispositions as well as field structures.
\end{abstract}

Keywords Cooling out $\cdot$ Career change $\cdot$ Habitus $\cdot$ Structure of professional motivation

\section{Cooling out dans le monde du travail : le changement de profession comme conséquence de l'inadéquation entre l'habitus et le champ}

Résumé Cet article présente les changements de profession sous l'angle du concept de cooling out. Ce faisant, ce concept fait l'objet d'un double élargissement. Premièrement, il n'est pas uniquement conçu, dans le prolongement de la discussion inspirée de Goffman, comme une stratégie de sélection dirigée de l'extérieur vers un sujet mais également comme une stratégie d'origine interne d'intervention en situation de crise pour gérer des déceptions professionnelles. Deuxièmement, le cooling out en milieu professionnel est conceptualisé en référence à Bourdieu comme conséquence de l'échec de la mise en adéquation des conditions de réussite professionnelle et des dispositions individuelles afin de dépasser l'opposition binaire entre auto-sélection et hétéro-sélection et de prendre en considération ce qui se joue entre le champ et le sujet. À partir d'études de cas empiriques conduites auprès de personnes ayant changé de profession, il est montré que les décisions de changer de profession ne peuvent être entièrement attribuées ni à l'un ni à l'autre mais représentent seulement l'aboutissement d'une accumulation continue d'expériences biographiques relevant autant des dispositions individuelles que de la structures des champs.

Mots-clés Cooling out · Changement de profession $\cdot$ Habitus $\cdot$ Structure des incitations professionelles 


\section{Einleitung}

Einen einmal eingeschlagenen Berufsweg zu verlassen, ist in einem relativ starr gegliederten Berufssystem in der Regel mit finanziellen und sozialen Nachteilen verbunden (vgl. Seibert 2007). Dennoch wechseln immer wieder Menschen, teils ohne äußerlich erkennbare Not, ihren Beruf, in Deutschland zwischen 1993 und 2008 etwas mehr als drei Prozent (Nisic und Trübswetter 2012). In der Statistik wird dabei zumeist nur zwischen unfreiwilligen und freiwilligen Wechseln unterschieden (vgl. ebd.). Doch im Berufsleben sind Freiwilligkeit und Unfreiwilligkeit oftmals schwer voneinander abzugrenzen, sodass die zu einem Berufswechsel führenden Gründe entlang dieser Dichotomie nur unzureichend erfasst werden können. Zwar wird im Zuge der Individualisierungsthese konstatiert, dass ,das Korsett institutioneller Regelungen seitens des Arbeitsmarktes, des Bildungssystems und des Sozialstaates [...] immer brüchiger geworden ist" (Schaeper et al. 2000, S. 84), aber damit sind nicht nur Gestaltungsmöglichkeiten, sondern auch Gestaltungszwänge verbunden, die nicht zwangsläufig als individuelle Bereicherung empfunden werden. Hinzu kommen herkunftsbedingte Prägungen, die gleichsam hinter dem Rücken der Akteure Einfluss auf die individuelle Lebensgestaltung nehmen (vgl. hierzu etwa Schelepa 2010).

Eine Möglichkeit, der Dichotomie von Freiwilligkeit und Unfreiwilligkeit bei der Erklärung von Berufswechseln ein Stück weit zu entkommen, bietet das von Erwing Goffman (1952) entwickelte Cooling-out-Konzept. Es beschreibt ein Bündel von Strategien, mit deren Hilfe Organisationen und Personen eine heikle Diskrepanz zwischen den an eine Person gerichteten Rollenerwartungen und ihrer Selbstwahrnehmung auf behutsame Weise aufzulösen versuchen, um bei der betreffenden Person eine Identitätskrise mit negativen Folgen für alle Beteiligten zu verhindern. Dazu zählen etwa das Offerieren von alternativen Selbstdeutungsangeboten und Handlungsmöglichkeiten, die der ,,auszukühlenden“ Person zu einem neuen, an die soziale Realität angepassten Selbstbild verhelfen sollen.

In der soziologischen Bildungs- und Karriereforschung werden Abbrüche von Bildungs- und Berufskarrieren bereits von etlichen Autor*innen als Ergebnis eines beruflichen Cooling out gedeutet (vgl. Kahlert 2011). Damit wird auf vielgestaltige soziale Prozesse innerhalb von Institutionen oder Organisationen verwiesen, die bei den Subjekten den Eindruck erzeugen, beruflich am falschen Platz zu sein und sie zu einer berufsbiographischen Umorientierung anstiften. So wird etwa das Phänomen der zahlreichen Karriereabbrüche von Frauen in hoch qualifizierten Berufsfeldern auf verschiedenartigste Praktiken der Abwertung und Ausgrenzung zurückgeführt und als letzte Möglichkeit der Selbstbehauptung interpretiert. Auf Basis einer Untersuchung über das Promotionswesen der Max-Planck-Gesellschaft berichten bspw. Allmendinger et al. (1999) über ein Bündel von Negativerfahrungen unter Doktorandinnen (z.B. geringe Ansprechmöglichkeiten ihrer Mentor*innen, langes Warten auf Rückmeldungen zu angefertigten Dissertationskapiteln, fehlende Themenabsprachen oder unklare berufliche Perspektiven), die den Wissenschaftlerinnen das Gefühl vermittelten, „nicht gewollt zu sein“ und ihr Interesse an einer wissenschaftlichen Tätigkeit ,auskühlen“ ließen. 
Im Feld der Wirtschaft werden Cooling-out-Prozesse vor allem unter Beschäftigten im mittleren und höheren Management beobachtet, die an der jeweiligen spezifischen Messlatte für Leistung scheitern (Miller und Robinson 2004; Nadai 2007). Dabei zeigt Nadai, dass ,cooling out nicht unbesehen mit Exklusion gleichgesetzt werden [darf], sondern [...] auch integrative Wirkung haben [kann]“ (ebd., S. 453). Im Rahmen von Organisationsfallstudien hat sie herausgefunden, dass Unternehmen vielfach bemüht sind, durch Herabsetzen von Leistungserwartungen ihrerseits eine Anpassung an das (mindere) Leistungsniveau einzelner Mitarbeiter vorzunehmen.

Dennoch wissen wir bisher wenig, was beim beruflichen Cooling out genau geschieht. Ist es etwa die Begeisterung für den Beruf bzw. für eine bestimmte Tätigkeit auf der Sachebene, die gleichsam zum „Auskühlen“ gebracht wird, weil sie nicht mit den Anforderungen des beruflichen Feldes übereinstimmt? Oder sind es Erwartungen ohne unmittelbaren Sachbezug wie das Bedürfnis nach Zugehörigkeit, Anerkennung oder Erfolg, die in diesem Prozess einer Revision unterzogen werden? Mit dem folgenden Beitrag möchte ich die Beweggründe von Berufswechseln genauer in den Blick nehmen. ${ }^{1}$ Das Cooling-out-Konzept dient dabei als Heuristik und soll das Augenmerk auf den Prozess der Transformation - oder, um im Bild zu bleiben, der Abkühlung von etwas, das einmal heiß oder warm gewesen sein muss richten, der einer Entscheidung zum beruflichen Wechsel in der Regel vorgelagert ist und dessen Entstehungszusammenhänge es zu rekonstruieren gilt. Im Unterschied zu Goffman (1952), für den Cooling out ein funktionalistisches Konzept darstellt, das den Prozess beschreibt, in dem Personen durch verschiedene Strategien mit einer Niederlage versöhnt werden (,,adaptations to loss“: ebd., S. 462), um deren destruktives Aufbegehren zu vermeiden oder abzuschwächen, wird davon ausgegangen, dass ein Cooling out nicht zwingend intendiert sein muss, sondern auch durch erlebte Missstände oder Enttäuschungen ausgelöst werden kann, die sich infolge einer unzureichenden Übereinstimmung zwischen den Bewährungsbedingungen eines beruflichen Feldes und individuellen Dispositionen einstellen. Dazu wird das Cooling-out-Konzept um eine habitustheoretische Perspektive ergänzt. Neben expliziten Selektionsstrategien sind damit zum einen auch die in den Strukturen von Berufsfeldern eingelassenen impliziten Selektionsmechanismen als mögliche Auslöser eines Cooling out von analytischem Interesse, etwa implizite Normen, Regeln, dominierende Deutungsmuster und kulturelle Praxen, die auf der Subjektebene als be- oder ausgrenzend erlebt werden können (vgl. Kahlert 2011), zum anderen die Dispositionen oder Habitusformationen der Subjekte, die gleichsam die andere Seite des misslungenen Passungsverhältnisses darstellen.

Ein berufliches Cooling out als Folge einer unzureichenden Übereinstimmung von Habitus und Feld zu denken, verlässt die Entweder-Oder-Struktur von Selbst- und Fremdselektion und ermöglicht, derartige Prozesse nicht nur als ein „Kaltstellen“ von Personen gegen deren Willen zu verstehen, sondern auch als eine spezifische Form der individuellen Krisenintervention, bei der die betroffene Person ein Mismatch zwischen den eigenen beruflichen Orientierungen (Giegel et al. 1988) und

\footnotetext{
1 Der Beitrag greift zurück auf Ergebnisse des DFG-Projektes „Cooling out als Transformation der beruflichen Antriebsstruktur“, das zwischen 2013 und 2017 am Wissenschaftszentrum Berlin für Sozialforschung durchgeführt wurde.
} 
den Feldbedingungen erkennt und verarbeitet. Die Entscheidung zum Berufswechsel stellt aus dieser Perspektive nur den End- und Umschlagspunkt eines solchen Prozesses dar, in dem die Akteur*innen ihre Deplatzierung erkennen und sich wiederum ganz im Sinne Goffmans (1952, S. 456) auf die Suche nach einem für sie passenderen Platz (,,a new framework in which to see himself“) begeben: „Sie mutet zwar wie eine Selbstselektion (als individuelle und autonome Entscheidung) an, ist aber gleichermaßen ein Produkt von Feldstrukturen und biographischen Erfahrungsaufschichtungen“ (Matthies und Rehbein 2020, S. 88). ${ }^{2}$

Ziel des Beitrags ist es, die ein berufliches Cooling out auslösende Konstellation freizulegen und herauszuarbeiten, wie darin Feldstrukturen und individuelle Dispositionen auf relationale Weise ineinandergreifen. Der Akzent liegt dabei auf der individuellen Wahrnehmung und Deutung von Arbeitsstrukturen und deren Übereinstimmung oder Nichtüberstimmung mit „eigenen Werten und Sinnansprüchen“ (Hardering 2015, S. 406). ${ }^{3}$ Methodisch dockt die Untersuchung an eine rekonstruktionslogische Vorgehensweise an, bei der Sinnansprüche genauso wie Möglichkeiten und Barrieren im beruflichen Feld aus berufsbiographischen Selbstbeschreibungen rekonstruiert werden.

Der Beitrag ist folgendermaßen aufgebaut: Zunächst erläutere ich die Implikationen einer Konzeptualisierung von Cooling out als Folge eines Mismatches von Habitus und Feld (Abschnitt 2). Anschließend stelle ich die Datenbasis und Methodik der Untersuchung vor (Abschnitt 3). Abschnitt 4 präsentiert sodann zwei prägnante Beispiele unterscheidbarer Cooling-out-Konstellationen, die einen Einblick in die Entstehungszusammenhänge von Berufswechseln und die diesbezügliche Rolle von Mismatches geben. In Abschnitt 5 werden die empirischen Befunde resümierend im Hinblick auf ihre empirische und theoretische Reichweite diskutiert.

\section{Cooling out im Kontext von Habitus und Feld}

Mit Willems fasse ich Habitus als sowohl bindende wie ermöglichende Verhaltensdispositionen, die sich ,,vor allem und lernschicksalhaft in primären Sozialisationsprozessen entwickeln und als ,zweite Natur" der Akteure tendenziell unbewusst (spontan, intuitiv selbstverständlich) fungieren“(Willems 1997, S. 182). Das schließt an Bourdieu an, der mit dem Habitusbegriff auf früheren Erfahrungen aufbauende „Wahrnehmungs-, Denk- und Handlungsschemata“ (Bourdieu 1993, S. 101) verbindet, die zugleich erklären, weshalb soziale Praktiken im Zeitverlauf eine hohe

\footnotetext{
2 Eine solche Auslegung von Cooling out findet sich auch in Arbeiten zum Bildungssektor, in denen die gesellschaftliche Erwartung einer normalen Bildungslaufbahn als subjektiver Interpretationsfilter von Jugendlichen identifiziert wird, der bei all jenen, die von dieser Erwartung abweichen, zu einer individuellen Anpassung der Leistungs- und Bildungsaspirationen an das ihnen möglich erscheinende führe (Heinz 1996; Solga 2004; Stauber et al. 2007). Die Jugendlichen selektieren sich demnach gewissermaßen selbst aus, um eine befürchtete Stigmatisierung als Dauerabweichler zu umgehen.

3 Einschlägige Hinweise auf Passungsprobleme zwischen beruflichen Orientierungen und Bedingungen im Berufsfeld liefern Untersuchungen zu beruflicher Identität und sinnvoller Arbeit (s. Hardering 2017 sowie Hardering und Will-Zocholl 2019) sowie zu dem vor allem unter Frauen beobachteten Phänomen des sogenannten Opting-out (etwa Mainiero und Sullivan 2005).
} 
Übereinstimmung und Konstanz aufweisen. Für Bourdieu leistet der Habitus folglich die Vermittlung zwischen Struktur und Praxis. Dabei ist er sowohl ein hervorgebrachtes Werk, ein opus operatum, das von den Akteur*innen in Relation zum sozialen Umfeld, in dem sie zu Hause sind, erworben wird, als auch ein stillschweigender Taktgeber, ein modus operandi, insofern er Praxisformen hervorbringt, die die Aufrechterhaltung gegebener sozialer Strukturen gewährleisten. Diese relationale Sichtweise findet sich auch in Goffmans Rahmenkonzept, in dem er darlegt, wie sich soziale Praxis über mehr oder weniger in Rahmen eingebettete ritualisierte Interaktionen entfaltet (vgl. Rehbein und Saalman 2014, S. 111; Willems 1997).

Mit Blick auf moderne Gesellschaften ist das Habituskonzept bei Bourdieu eng mit dem Begriff des sozialen Feldes verknüpft, über den er den Prozess der gesellschaftlichen Ausdifferenzierung in relativ autonome soziale Bereiche beschreibt (vgl. Jurt 2010, S. 14). Dabei ist ein Feld durch eine je eigene Logik, eigene Regeln und eine eigene ,illusio“ bestimmt, in deren Relation auch feldspezifische Habitus (re-)produziert werden. Bourdieu veranschaulicht das Zusammenspiel von Habitus und Feld gern am Beispiel des Spiels, weil sich daran sehr gut erkennen lässt, wie der Habitus - hier als „Sinn für das Spiel“ bezeichnet - ermöglicht, dass die Spieler*innen eine vorausschauende Haltung zu den Erfordernissen des Spiels einnehmen (Bourdieu 2014, S. 122). Ein guter Fußballspieler ist bspw. ,immer dort, wo der Ball im nächsten Augenblick hinkommt. Er weiß, wie er sich zu bewegen hat, er kennt die zahllosen Tricks und Kniffe, die gerade noch erlaubt sind und den entscheidenden Vorteil gegenüber den Gegenspielern verschaffen“ (Rehbein und Saalman 2014, S. 112).

Auch die einem sozialen Feld innewohnende Dynamik lässt sich am Spiel gut erkennen. Nicht nur, dass die Spieler unterschiedliche Aufgaben wahrzunehmen haben, sie verfolgen auch divergierende Interessen und nehmen innerhalb der Mannschaft unterschiedliche Positionen ein. „Es ist ein ,Spiel“ um Macht und Einfluss, um die Durchsetzung der eigenen Sichtweise im Horizont des Möglichen“, so Beate Krais (2000, S. 39). Jeder, der sich auf dieses Spiel einlässt, muss den Glauben daran haben - die ,illusio“, wie Bourdieu es nennt -, „daß das, was in einem sozialen Spiel geschieht, einen Sinn hat, und daß das, was bei ihm auf dem Spiel steht, wichtig und erstrebenswert ist" (Bourdieu und Wacquant 1996, S. 148). Je besser die Akteur*innen sich die Regeln des Feldes einverleibt haben, umso geschickter und gewinnbringender können sie im Feld agieren und ihre Position ausbauen (vgl. Lenger et al. 2013, S. 32). Allerdings dürfen sie den Bogen dabei auch nicht überspannen und das Spiel gefährden.

Übertragen auf berufliche Felder folgt daraus, dass die dort geltenden Regeln und Logiken von den Akteur*innen inkorporiert sein müssen. Dies gilt umso mehr, je mehr Spielraum eine konkrete berufliche Position beinhaltet (Bourdieu 1997, S. 202, 207). Gleichwohl ist davon auszugehen, dass die dazu notwendige Anpassung des individuellen „Primärhabitus“ an die Erfordernisse der beruflichen Position nie vollständig gelingt. Ein gewisser Mismatch zwischen Habitus und Feld ist folglich eher der Normalfall. „Der Habitus“, so Bourdieu (1997, S. 206), ,ist weder notwendigerweise angemessen noch notwendigerweise kohärent. Er verfügt über Integrationsstufen, die vor allem ,Kristallisationsstufen " des eingenommenen Status entsprechen“. Und nicht zuletzt können Dispositionen bedingt durch Veränderungen 
im Feld einer Art „Verschleiß“ unterliegen, wie Bourdieu am Beispiel der algerischen Arbeiter erwähnt, deren „Dispositionen einer präkapitalistischen Welt nicht den Anforderungen einer westlich-kolonialisierten Welt entsprachen“ (Jurt 2010, S. 15). Dieses Risiko einer unvollkommenen oder gar misslungenen (Um-)Programmierung des Habitus dürfte in der gegenwärtigen Arbeitsgesellschaft, in der die spezifischen beruflichen Anforderungen einer unaufhörlichen Revision unterworfen sind, ein kontinuierliches Problem darstellen. Es ist deshalb damit zu rechnen, dass die Zahl der Akteur*innen, die sich auf ihrer Position deplatziert fühlen, erheblich zugenommen hat. Statt ,den Habitus den Erwartungen des Postens“ (Bourdieu 1997, S. 202) anzupassen, könnten diese Akteur*innen den „Glauben an das Spiel“ verlieren und ihr Commitment gegenüber dem beruflichen Feld sukzessive aufkündigen.

Um die Entstehungszusammenhänge derartiger Auskühlungsprozesse aus der Habitus-Feld-Perspektive zu rekonstruieren, sind folglich die spezifischen Formationen und wechselseitigen Bedingtheiten zu identifizieren, die für das das Cooling out auslösende Mismatch verantwortlich zeichnen. Mit Blick auf die Akteur*innen geht es dabei vor allem um jene Dispositionen, die deren beruflichem Handeln strukturierend zugrunde liegen: Was etwa hat sie bewogen, genau diesen Beruf zu ergreifen? Welche expliziten und latenten Erwartungen waren damit verbunden? Wie haben sie das Berufsfeld wahrgenommen und was hat sie zum beruflichen Wechsel angestiftet? Diese Momente des Habitus fasse ich in Anlehnung an Thomae (1944) als berufliche Antriebsstruktur.

Mit dem Begriff des Antriebs bringt Thomae die materiale Tatsache zum Ausdruck, ,daß alle unsere psychischen Funktionen und motorischen Äußerungen durch irgendeine Kraft hervorgebracht (angetrieben) sein müssen“ (Bostroem 1928 zit. nach Thomae 1944, S. 24). Folgerichtig umschließt der Antrieb alle irgendwie auf Handlungen abzielenden Phänomene wie Drang, Affekt, Trieb, Sucht, Wunsch, Streben, Interesse, Wollen, Begehren etc. ${ }^{4}$ Deren energetischer Charakter gründet für Thomae in der „Frontstellung zur Welt als Widerstand“ (Thomae 1944, S. 76), wobei die „Spannung zwischen Gegenwart und Zukunft“ (Thomae 1944, S. 53) eine besondere Rolle spielt. Für das Subjekt stellt sich fortwährend die Frage: Was kann (und will) ich tun, um meinem Leben Gestalt zu geben? Je nach Lebenslage kann dieser Entscheidungszwang verschiedene Kräfte in Bewegung setzen und folglich auch ganz unterschiedlich empfunden werden. Denn als Teil des Habitus ist die Antriebsstruktur an Raum und Zeit gebunden, weil ,fast alles, was Form und geronnene Struktur am menschlichen Charakter ist, einmal Geschehen war und [...] vieles, was jetzt Geschehnis ist, einmal Form, Haltung, Bereitschaft, Anlage, Triebkraft werden kann“" (Thomae 1955, S. 1).

Damit rückt die Genese der Antriebsstruktur in den Fokus. Neben der Frage, inwiefern sich typische Mismatch-Konstellationen von Feldverfassungen, Habitus und beruflicher Antriebsstruktur identifizieren lassen und wie sich das auf die Antriebsstruktur auswirkt und ggf. Cooling-out-Prozesse in Gang setzt, ist auch von

\footnotetext{
${ }^{4}$ Zur Mannigfaltigkeit und Differenzierung der Antriebe siehe Thomae 1944, S. $153 \mathrm{ff}$.

${ }^{5}$ Das hat Berührungspunkte mit der Bewährungstheorie von Ulrich Oevermann (1995), wenngleich Thomaes Überlegungen säkularisierter daher kommen als in dem auf Max Webers protestantische Ethik zurückgehenden Bewährungsbegriff.
} 
Interesse, welche primär- und sekundärsozialisatorischen Prozesse die jeweilige Antriebsstruktur gleichsam geformt haben.

\section{Datengrundlage und Methodik}

Empirische Grundlage der im Folgenden präsentierten Befunde zur Entstehung eines beruflichen Cooling out sind 22 biographisch-narrative Interviews, die zwischen 2015 und 2017 erhoben wurden. Die Fallauswahl orientierte sich an den Kriterien des theoretischen Samplings (Glaser et al. 2010; Kelle und Kluge 1999) und zielte neben einer Repräsentation beider Geschlechtsgruppen - insgesamt wurden zwölf Frauen und zehn Männer interviewt - auf eine maximale Fallkontrastierung im Hinblick auf die das Cooling out auslösenden Faktoren, soweit sich das im Zuge der Vorgespräche eruieren ließ.

Die Interviewpartner*innen wurden etwa zu gleichen Teilen über Mundpropaganda (12 Fälle) und einen Flyer (10 Fälle) akquiriert, der über berufliche Netzwerke wie XING oder LinkedIn und berufliche Beratungs- und Coachingeinrichtungen verbreitet wurde. Der Flyer richtete sich explizit an Menschen, die aufgrund zunehmender Unzufriedenheit mit ihrer beruflichen Tätigkeit einen beruflichen Wechsel erwogen oder bereits vollzogen hatten. Im Zuge der Erläuterung des Projektzusammenhangs wurde dieser Prozess auch als „Cooling out des beruflichen Antriebs“ bezeichnet. Ein großer Teil der Interviewten hat sich demzufolge also selbst als Cooling-out-Fall adressiert und dabei möglicherweise eine Ex-post-Rationalisierung des Berufswechsels vollzogen. ${ }^{6}$ Diesem Umstand der Selbstselektion mag auch geschuldet sein, dass alle Fälle eher erfolgreiche Karriereverläufe repräsentieren und nicht im klassischen Sinn beruflich gescheitert sind.

Mit einer Ausnahme verfügen alle Interviewteilnehmer*innen über einen berufsqualifizierenden Abschluss; die Mehrzahl (18 Fälle) hat zudem einen Hochschulabschluss. Die repräsentierten Berufsfelder sind divers, allerdings haben soziale Berufe ein leichtes Übergewicht. Das Spektrum der Geburtsjahrgänge reicht von Jahrgang 1948 bis 1983 bei den Frauen und von 1950 bis 1971 bei den Männern; alle Interviewten verfügen also über eine hinreichende Zeitspanne an Berufserfahrung.

Dem biographischen Ansatz der Studie entsprechend hob die Eingangsfrage der Interviews darauf ab, beim Gegenüber ein möglichst zusammenhängendes (berufs-) biographisches Narrativ zu evozieren. Mit Bezug auf den jeweils stattgefundenen Berufswechsel wurde das Interview mit folgender Frage eröffnet: Bevor wir darüber sprechen, weshalb Sie den Beruf aufgegeben haben, interessiert uns, wie Sie überhaupt dahin gekommen sind. Was hat Sie bewogen, diesen Beruf zu ergreifen? Die Interviewten wurden also als Subjekte adressiert, die auf eine eigene Berufsbiographie zurückblicken, was zumeist eine längere autobiographische Erzählung auslöste.

\footnotetext{
${ }^{6}$ Explizit formuliert das eine Interviewpartnerin, die über den Flyer auf das Projekt aufmerksam wurde und sich daraufhin per E-Mail an uns wandte. Sie schrieb: „Ich hatte spontan den Eindruck, Sie suchen mich! Seit 2012 befinde ich mich in einer beruflichen Umbruchsituation: von 29 Jahren bei der Firma [Name] hin zum Start in die Selbstständigkeit dieser Tage. Und über das Phänomen ,Cooling out ' habe ich mir in den letzten Jahren viele Gedanken gemacht, da selbst stark betroffen - ich hatte nur noch keinen Namen dafür. Aber ich brannte halt nicht mehr“.
} 
Rückfragen zu konkreten biographischen Ereignissen oder Wendepunkten wurden erst nach Ende der ersten Narration gestellt. Im Anschluss an den narrativen Teil wurden mittels eines standardisierten Fragenkatalogs Daten zur Herkunftsfamilie wie Geburtstag(e), Beruf(e) und Lebensort(e) sowie zum Lebenslauf des/der Interviewten, zu Veränderungen in der Familienkonstellation sowie zu Partnerschaften und Kindern erhoben, die die lebensgeschichtliche Kontextuierung des Interviewnarrativs methodisch absichern sollten.

Die Interviews fanden überwiegend in der Wohnung des/der Interviewten statt und dauerten in aller Regel zwischen 90 und 120 Minuten. Sie wurden digital aufgezeichnet und im Zuge ihrer Transkription anonymisiert. Die Analyse der verschriftlichten Interviewprotokolle erfolgte mittels einer Kombination aus Sequenzund Inhaltsanalyse. Zunächst wurde die Eingangssequenz des Interviewprotokolls im Sinne der objektiven Hermeneutik einer Feinanalyse unterzogen. Dazu wurden die im Interviewprotokoll enthaltenen Äußerungs- und Handlungsverläufe als eine Abfolge von Selektionen betrachtet (Oevermann 1991, S. 270), die Aufschluss auf die habituellen Dispositionen ihrer Urheber geben können. Die an das Material gerichteten Fragen lauteten an jeder Sequenzstelle aufs Neue (vgl. Fischer 2009, S. 28): Für welche der je gegebenen Handlungsoptionen entscheidet sich der/die Interviewte (Selektion)? Auf welche Weise geschieht das (Habitus)? Wie werden die Entscheidungen begründet (Deutungsmuster)? Zug um Zug wurden so die dem Denken und Handeln eines Individuums zugrunde liegenden und den Lebensverlauf strukturierenden Prinzipien sowie deren Genese und ggf. Transformation aus dem Material rekonstruiert. Um das bekanntermaßen aufwendige Verfahren der Sequenzanalyse abzukürzen, wurden außer der Eingangssequenz nur noch solche Textpassagen einer Feinanalyse unterzogen, die kritische Episoden thematisieren. In ähnlicher Weise wurden auch die im Rahmen eines jeden Interviews erhobenen biographischen Daten daraufhin befragt, inwieweit an bestimmten biographischen Schnittstellen kritische Lebensphasen erkennbar sind und was darin zum Ausdruck gebracht wird. In der Gesamtschau wurden zwei Blickrichtungen aufgespannt: Zum einen wurde der reale Verlauf der erzählten Lebensgeschichte als ein auf der Bewältigung von Krisen beruhender „Prozess der Gestaltung einer offenen Zukunft“ (Oevermann 2009, S. 44) im Hinblick auf seine grundlegende Struktur sowie auf Anzeichen einer Strukturtransformation analysiert. Zum anderen wurde ausgehend vom beruflichen Cooling out rückblickend in die Vergangenheit gefragt, wie sich erklären lässt, dass die Biographie diese Form angenommen hat und welche Rückschlüsse sich daraus im Hinblick auf die Strukturen beruflicher Felder ziehen lassen. Die Analyse eines Interviews galt als abgeschlossen, wenn sich die Hypothese zur Struktur des Falls an weiteren Stellen des Protokolls bestätigte und im weiteren Durchgang durch das Material keine falsifizierenden Stellen aufgefunden wurden. Die Analyseergebnisse wurden fallbezogen dokumentiert und in Anlehnung an Schmeiser (2003) durch ein ausführliches Lebensereignisprotokoll ergänzt.

In den Fallrekonstruktionen zeigte sich ein deutlicher Zusammenhang zwischen beruflicher Antriebsstruktur und Mismatch-Erfahrungen. Davon werden nachfolgend zwei Varianten präsentiert. Sie sind das Ergebnis einer typologischen Zuspitzung des empirischen Materials im Sinne einer Gruppierung der Fälle nach den Prinzipen 
größtmöglicher Homologie und gleichzeitig größtmöglicher Abgrenzung zueinander (Gerhardt 2001; Giegel et al. 1988; Kelle und Kluge 1999). ${ }^{7}$

\section{Cooling-out-Konstellationen}

In der folgenden Darstellung der beiden Konstellationen wird zunächst jeweils kurz erläutert, woran sich das Mismatch im Wesentlichen festmacht. Anschließend werden am Beispiel von prägnanten Fällen der Cooling-out-Prozess sowie die Ausprägung der Konstellation im Hinblick auf typische Merkmale der Antriebsstruktur, biographische Prägungen, Handlungsstrategien, markante Eckpunkte der beruflichen Werdegänge und konkrete Erfahrungen der Nichtpassung näher beschrieben.

\subsection{Karriereaspirationen kollidieren mit Karriererisiken - Cooling out als Scheiternsprävention}

In der ersten Konstellation haben wir es mit Personen zu tun, bei denen sich das Cooling out an einer unzureichenden Übereinstimmung von Karriereaspirationen, Risikobereitschaft und wahrgenommenen Karrierechancen im erwählten Beruf entzündet. ${ }^{8}$ Die berufliche Antriebsstruktur dieser Fälle ist durch eine relativ klare Erfolgsorientierung bestimmt, in der ein latentes Motiv des Überbietens elterlicher Karriereerfolge erkennbar ist. Die Fälle starten ihren Berufsweg typischerweise mit klaren Vorstellungen, geraten aber im Laufe ihrer beruflichen Entwicklung in Zweifel, ob ihre ambitionierten beruflichen Ziele unter den im Berufsfeld wahrgenommenen wettbewerblichen Bedingungen einer erfolgreichen Karriere auch erreichbar sind.

Prototypisch für diese Konstellation sind zwei Wissenschaftler*innen, der Biologe Lars Lambrecht und die Physikerin Claudia Ruprecht ${ }^{9}$, die herkunftsbedingt für eine wissenschaftliche Karriere eigentlich beide sehr gut ausgestattet sind. Schon die Eltern und zum Teil auch die Großeltern haben eine akademische Ausbildung absolviert und sind in gehobenen beruflichen Positionen tätig, sodass es in beiden Fällen an kulturellem Kapital (Bourdieu 1992) nicht mangelt. Für Claudia Ruprecht war ,das akademische Leben schon immer was sehr Konkretes“ ${ }^{\text {“ }}$, wo sie sich ,auch sehr gut vorstellen konnte in Zukunft zu arbeiten“. Und Lars Lambrecht hat bereits mit ,sieben, acht so was“ auf die Frage, was er einmal werden möchte, geantwortet: „Wissenschaftler!“”

\footnotetext{
7 Zentrale Vergleichs- bzw. Unterscheidungskriterien waren dabei die berufliche Antriebsstruktur und die jeweiligen Mismatch-Erfahrungen. Auf die Darstellung einer prägnanten dritten Variante, in der das Cooling out stark mit Somatisierungserscheinungen verwoben ist, muss hier aus Platzgründen verzichtet werden.

${ }^{8}$ Die Darstellung dieser Konstellation greift teilweise auf eine bereits erfolgte Veröffentlichung zum Ausstieg aus der Wissenschaft zurück (Matthies und Rehbein 2020).

9 Die Namen wurden aus Anonymisierungsgründen geändert.

${ }^{10}$ Die Zitate aus den Interviews wurden zwecks besserer Lesbarkeit behutsam sprachlich geglättet.
} 
Bis zum Eintreten der beruflichen Krise beschreiben beide Fälle ihren Werdegang als „sehr geradlinig“ und stellen damit nicht nur ihre klare Zielorientierung, sondern auch ihr intellektuelles Potenzial und ihre Leistungsfähigkeit heraus. Nach der Grundschule wechseln sie wie selbstverständlich auf das Gymnasium, machen dort ein Einser-Abitur und beginnen anschließend ein wissenschaftliches Studium. Lambrecht, dessen Interesse an der Wissenschaft „,schon immer“ mit Naturwissenschaft verknüpft war, entscheidet sich für Biochemie und grenzt sich damit vom alltagsweltlichen Bezug der elterlichen Berufe $a b$ - vor allem von dem der Mutter, die er als „Feld-Wald-Wiesenbiologin“ bezeichnet. Er möchte stattdessen ein Wissenschaftler werden, der sich für die ,allerkleinsten Zusammenhänge“ interessiert. Ruprecht beginnt ein Studium der Physik. Das Fach hat sie mit Fünfzehn während eines halbjährigen Auslandsaufenthaltes der Familie in den USA für sich entdeckt, wo sie der Physikunterricht, den sie bisher nur langweilig fand, ,einfach gepackt (hat)“. Dieses Erweckungserlebnis verschafft ihr endlich auch eine konkrete Vorstellung davon, wie sie die stumme Erwartung der Mutter, Professorin zu werden, mit Inhalt füllen kann. Ihr Interesse gilt der Teilchenphysik: „das zu verstehen im Prinzip, was die Welt im Innersten zusammenhält".

Mit Beginn des Studiums zeigen sich bei beiden deutliche Züge einer strategischen Optimierung ihrer Biographie. Sie studieren in Regelstudienzeit oder unterbieten diese sogar, absolvieren ,,aus lebenslauftechnischen Gründen“ (Lambrecht) Praktika und Zusatzkurse in international renommierten wissenschaftlichen Einrichtungen und beginnen nach Abschluss des Studiums umgehend mit der Arbeit an der Dissertation. Auch bei der Wahl der Promotionsstellen spielt das Renommee der Institution eine entscheidende Rolle. So ist für Lambrecht das ,hochkarätige Institut" wichtiger als das Thema, welches ihm ,vorgeschlagen“"wurde. Und Ruprecht setzt alle Hebel in Bewegung, um ihre Promotion an jener namhaften internationalen Forschungseinrichtung anfertigen zu können, an der sie schon als Praktikantin tätig war und an der auch ihr kürzlich geehelichter Mann, ebenfalls ein Physiker, arbeitet.

Erste Gefühle von Mismatch kommen bei beiden in der Promotionsphase auf, allerdings aus unterschiedlichen Anlässen. Bei Lambrecht ist es die Wahrnehmung, den neuerdings in der Wissenschaft geforderten Dispositionen (siehe Matthies et al. 2015) - insbesondere dem Anspruch an die performative Inwertsetzung von Leistung, wie sie etwa in Forschungen zur Subjektivierung von Arbeit (Moldaschl 2003), zum unternehmerischen Selbst (Bröckling 2007) oder zu beruflich verwertbarem affektiven Kapital (Penz und Sauer 2016) beschrieben werden - nicht zu entsprechen. Dabei ist Lambrecht durchaus bereit, entgrenzt zu arbeiten und alle anderen Lebensbereiche dem angestrebten Ziel einer Professur unterzuordnen. Dennoch scheitert er - zumindest dem eigenen Eindruck nach - an den neuen Anforderungen an eine wissenschaftliche Persönlichkeit. Auf Konferenzen schafft er es nicht, Aufmerksamkeit und Interesse zu generieren, sondern steht zumeist im Abseits, wie er sagt: „Dass wirklich mal eine Traube von Menschen vor meinem Poster angehalten [...] und gesagt hat, ,ach das ist ja spannend', ja solche Erlebnisse sind einfach ausgeblieben“. Laut Selbstbeschreibung hat er kein Gespür für innovative Themen und findet so in der wissenschaftlichen Community kaum „Response“: „Ich hab’ auch gezielt, also mit wirklich schlafwandlerischer Sicherheit [...] immer Projekte rausgesucht, die ich total spannend fand und der Rest der Welt total langweilig“. 
Vor dem Hintergrund dieser Eindrücke realisiert Lambrecht im Laufe der Promotion schmerzhaft, dass es für eine erfolgreiche Karriere in der Wissenschaft nicht ausreicht, ein Thema mit Fleiß und Sachverstand zu bearbeiten. Dabei misst er sich an einem Kollegen, den er in vergeschlechtlichter Diktion als ,toller Hecht“ oder „golden boy“ bezeichnet, sowie an all jenen, die „brennender von ihrem Thema erzählen“, die „,interaktiv“ sind, die „Tatendrang“ aufweisen und forsch vorangehen, und ruft damit gleichsam jenes Bündel an ihm fehlenden Dispositionen auf, das Penz und Sauer (2016, S. 77) als ,affektives Kapital“ bezeichnen. Damit zielen sie auf „das Potenzial einer Person [...], andere Menschen zu affizieren, sie anzusprechen, sie zu berühren bzw. anzurühren, ihre Aufmerksamkeit zu erregen" (ebd.). Affektives Kapital als eine spezifische Form des Bourdieu'schen kulturellen Kapitals wird in Interaktionen aktiviert und ermöglicht die Bildung von sozialem Kapital (vgl. ebd.), und genau diese Aktivierung und Konversion gelingt Lambrecht nicht. Mit seinen Kollegen am Institut ist er kaum vernetzt und von seiner Chefin fühlt er sich zu wenig beachtet. Zunächst empfindet er das als ,unfair“, doch im Laufe der Zeit beginnen Selbstzweifel an seiner Eignung für die Agora des wissenschaftlichen Feldes. Ihm wird zunehmend gewahr, dass er das (neue) Ideal des Feldes, intrinsische Motivation, Kreativität und Begeisterung auch performativ in Szene zu setzen und „,neue Impulse [zu] entwickel[n]“, praktisch nicht erfüllen kann. Seine eigene Haltung zur Arbeit charakterisiert er demgegenüber relativ offen als extrinsisch motiviert: „Das war eine Aufgabe, die ich zu bewältigen hatte [...] was man von außen gegeben bekommt und bei der man erfolgreich ist oder nicht". Mit dieser Selbsteinschätzung wird das wahrgenommene Mismatch als ein persönliches Defizit (an-)erkannt und mündet in der Einsicht: „Da kann ich nicht wirklich mithalten“. Lambrecht schließt die Promotion zwar noch ab, befasst sich gedanklich aber mit einem Ausstieg aus der Wissenschaft und bewirbt sich auf vakante Stellen außerhalb der Forschung. Als er schließlich an einer großen deutschen Universität eine Stelle als Forschungsreferent findet, ist für ihn „,völlig klar: ich bleibe nicht“.

Bei Ruprecht kommt es zu ersten Erfahrungen von Mismatch im Zuge der Realisierung ihres Wunsches nach vielen Kindern, den sie mit ihrem Mann teilt. Vier Kinder sollen es werden, wie in den Familien, in denen beide groß geworden sind. Die familiäre Reproduktion gehört für Ruprecht wie für ihren Partner zu einem gelungenen Leben dazu. Zwei Kinder kommen in der Promotionszeit zur Welt, wobei die zweite Schwangerschaft mit der Fertigstellung der Dissertation zusammenfällt. Sie wird mit magna cum laude benotet und erreicht damit nicht das Niveau von Ruprechts bisherigen Abschlussnoten. Darauf angesprochen sagt sie: „Ich hab' die Doktorarbeit abgegeben eine Woche bevor mein zweites Kind geboren wurde, und ich hab' mich mit dem Aufschreiben der Arbeit sehr schwer getan. Und ich hab' die Doktorprüfung gemacht, da war der Kleine drei Monate alt und ich hab' 'ne ziemlich schlechte Prüfung hingelegt". Die Dissertationsnote betrübt sie allerdings nicht allzu sehr, da sie bereits vor dem Abschluss der Promotion ein zweijähriges Research Fellowship ihres Instituts erhalten hat, das in ihren Augen ein größerer Beleg für Exzellenz ist: „Das wiegt viel mehr [...], das ist eine Auszeichnung, das zu bekommen", sagt sie.

Richtig genießen kann Ruprecht die zwei weiteren Jahre am Institut jedoch nicht, denn die Frage, wie Familienbildung und wissenschaftliche Karriere zusammenge- 
bracht werden können, existiert weiterhin. Konkret äußert sich das Problem darin, dass ihr Ehemann sich angesichts der nunmehr zwei Kinder auf die Suche nach einer unbefristeten Anstellung begeben hat und schließlich in einer anderen Stadt außerhalb der Wissenschaft fündig geworden ist. Beiden ist damit klar, dass dieser Ort auch der künftige Lebensmittelpunkt der Familie sein soll. Sie kaufen dort ein Haus, richten es nach ihren Wünschen her und nach Beendigung des Fellowship zieht auch Ruprecht mit den beiden Kindern in das neue Heim.

Während die Familie nunmehr wieder zusammenleben kann, nimmt für Ruprecht die Verunsicherung hinsichtlich ihrer beruflichen Zukunft zu. Zwar findet sie am neuen Wohnort sofort eine Fünfjahresstelle an einem ebenfalls renommierten Forschungsinstitut und kann dort ihre wissenschaftlichen Arbeiten fortsetzen, doch mit der dritten Schwangerschaft, die kurz darauf beginnt, fragt sie sich: „Wo soll's denn jetzt eigentlich hingehen und was kommt dann danach?" Für das Ziel, Professorin $\mathrm{zu}$ werden, rechnet sie sich aufgrund der familialen und räumlichen Bindung inzwischen ,ganz schlechte Chancen“ aus. Dabei sind ihre Überlegungen wie bei Lambrecht von dem Eindruck getragen, mit den anderen nicht mithalten zu können; als Vergleichsfolie hat auch sie einen männlichen Wissenschaftler im Auge. Sie misst sich an einem Kollegen, der ,,jeden Tag im Büro zehn Stunden sitzt und natürlich eine ganz andere Publikationsliste (hat)“, und fürchtet, dass sie im Wettkampf um die wenigen vakanten Professuren am Ort gegen solche Konkurrenten ohne Zusatzbelastung durch Care-Arbeit chancenlos ist. Am Ende ihrer Überlegungen und Zweifel kommt sie zu dem Schluss, dass es keinen Sinn hat, weiter an diesem Wettbewerb teilzunehmen: „Bevor ich mit den inzwischen drei Kindern wirklich ewig arbeite, 'nen richtigen Stress mache und dann irgendwann mit knapp vor 40 feststelle, hat doch nicht geklappt, lass ich's lieber bleiben und geh 'nen anderen Weg“" Schon im Zuge des Ortswechsels hatte sie sich parallel auch in der Industrie beworben, war dort aber erfolglos. Nach Entbindung des dritten Kindes unternimmt sie über den Weg einer Jobmesse einen erneuten Anlauf und bekommt bei einem Ingenieurdienstleister eine unbefristete Stelle angeboten, die sie nach kurzem Überlegen annimmt.

Für Lambrecht wie für Ruprecht wurde der Cooling-out-Prozess durch die Erfahrung ausgelöst, dass es zwischen ihrem ambitionierten Karriereziel und den Erfolgsaussichten im beruflichen Feld eine große Ungewissheitszone gibt, auf die sie sich nicht einlassen wollten. Dabei spielt weniger eine Rolle, ob die beiden ihre Karrierechancen realistisch eingeschätzt haben, als der Umstand, dass sie für den Umgang mit dem Hasard des wissenschaftlichen Berufs habituell offensichtlich nicht gut genug ausgerüstet sind. Diese Wahrnehmung hat bei beiden zu einer erheblichen persönlichen Krise geführt, die erst nach Auffinden einer für sie versöhnlichen beruflichen Alternative bewältigt werden konnte. Eine wichtige Rolle spielte dabei das Finden eines Narrativs, mit dem sie das Scheitern an den eigenen Ansprüchen zugleich als Stärke beschreiben konnten - der Stärke nämlich, dass sie selbst es waren, die zu einer realistischeren Einsicht ihrer Möglichkeiten gelangt sind. Lambrecht, der mit der festen Überzeugung in die Wissenschaft gestartet ist, ,in der akademischen Laufbahn (zu) bleiben“, trifft die Erkenntnis, in diesem Feld keine Anerkennung zu finden, ausgesprochen hart und lässt ihm seinen eingeschlagenen Berufsweg sinnlos erscheinen. Er fragt sich: „Was mache ich hier? Weshalb bin 
ich Biochemiker und promoviere, wenn das Ergebnis keine Professur sein soll? [...] Was mache ich denn dann mit meinem Leben?" Im Lauf dieser Auseinandersetzung gelangt er zu der Erkenntnis, dass sein eigentliches Interesse gar nicht das Erzeugen, sondern die Rezeption von (bereits bestehendem) Wissen ist: „Ich hab' gemerkt, mir reicht es, über die Dinge zu erfahren [...], an einem guten Seminar teilzunehmen und einem spannenden Vortrag zuzuhören oder ein Paper zu lesen, das gut und spannend geschrieben ist", beschreibt er diesen Prozess der gedanklichen Umorientierung. Am Ende sucht er systematisch nach beruflichen Alternativen, die bei ihm einen Reiz auslösen. Er recherchiert nach „großen Organisationen“ und „großen Initiativen“ sowie „diesen ganzen supranationalen Sachen“ und fragt sich: „Könnte ich mich hier sehen?" Das Renommee seines potenziellen Arbeitgebers scheint ihn in gewissem Maße mit einer abgebrochenen Wissenschaftskarriere zu versöhnen und das Versprechen zu bergen, im Zentrum relevanter, beachteter und sozial anerkannter Arbeit zu stehen, anstatt kaum wahrgenommen in der wissenschaftlichen Peripherie zu verbleiben. Als gelöst empfindet er die Krise, nachdem er für seine Zukunft „diesen Begriff Wissenschaftsmanagement gefunden und definiert hatte". Damit war es ihm möglich zu ,differenzieren zwischen ,ich will selber die Wissenschaft vorantreiben oder das wissenschaftliche Umfeld gestalten““ und ,plötzlich [zu merken], ,ja, das ist das wissenschaftliche Umfeld, das ich gestalten will““.

Hier zeigt sich, dass ein erfolgreich bewältigtes Cooling out mit dem Erwärmen für eine Alternative oder - allgemeiner gefasst - mit dem Finden eines neuen Selbstbildes einher geht, worauf bereits Goffman (1952) hingewiesen hat. Deutlich kommt dieses Zusammenspiel auch im Fall von Ruprecht zum Ausdruck. Als sie das Ausmaß der Ungewissheit eines Karriereerfolgs in der Wissenschaft sowie die damit verbundenen Einschränkungen für ihr privates Leben realisiert, freundet sie sich mit den Vorzügen eines geregelteren Berufslebens außerhalb der Wissenschaft an. Der Janusköpfigkeit von geistiger Autonomie und Unsicherheit, Internationalität und Mobilität im „,akademischen Leben“ stellt sie ein Modell des „guten Lebens“ mit eher traditionell anmutenden Werten der Familie - ,also ich würd' jetzt nicht die Kinder gegen 'ne wissenschaftliche Karriere tauschen", sagt sie -, der sozialen Integration und materiellen Annehmlichkeiten entgegen. Sie vergleicht ihre Freundeskreise, den alten, akademischen, in dem sich viele Gespräche um Existenz- und Zukunftssorgen drehen - ,immer wie geht's weiter, für alle auf 'nem ganz existenziellen Level“"-, mit dem neuen, der sich vor allem aus Kolleg*innen ihres Mannes zusammensetzt und wo „man [...] sich darüber (unterhält), wer am Wochenende im Konzert war oder wo man gut mit den Kindern 'nen Ausflug hinmachen kann und weiß ich nicht. Dann gibt's welche, die haben ihr Boot oder einer ist glaub' ich Segelflieger, und das sind so die Themen, man erzählt sich sowas und man hat Hobbys, die man entwickelt". Parallel reflektiert sie ihre Erfahrungen in der Wissenschaft und kommt ganz ähnlich wie Lambrecht zu dem Ergebnis, dass das, was ihr an der Arbeit Freude macht, nicht die wissenschaftliche Tätigkeit im engeren Sinn ist, sondern das Arbeiten im Team und die praktischen Tätigkeiten, das Organisieren und Koordinieren. „Das Geschriebene“, resümiert sie, „,spricht nicht so dolle zu mir“. Vom Zauber, den die Teilchenphysik noch als Teenager auf sie ausgeübt hatte, ist nun keine Rede mehr. Stattdessen wird der ursprüngliche Anspruch auf ein 
umfassendes Verständnis der Welt zu einem Interesse an überschaubaren Aufgaben und am Lösen von alltagspraktischen Problemen umgedeutet.

Mit Goffman (1952) und an ihn anschließend Clark (1960) ließen sich diese Cooling-out-Fälle für Felder mit hochselektiven Karrierebedingungen, wie es in der Wissenschaft der Fall ist, als typisch einordnen. Im Fall von Lambrecht wird das Cooling out vor allem infolge von Passungsproblemen ausgelöst, die aus den veränderten Arbeitsbedingungen im Zuge des institutionellen Wandels (Jansen 2007; Matthies und Torka 2019) resultieren und zu einer Umwertung dessen führen, was als symbolisches Kapital anerkannt wird. So sieht Lambrecht sich zwar als hoch motivierten Forscher, doch die neue Erwartung an die performative Inwertsetzung von Leistung kann er nicht erfüllen. Eine erfolgreiche wissenschaftliche Karriere erscheint ihm deshalb aussichtslos. Im Fall von Ruprecht erwachsen die Probleme der Passung aus dem Grundkonflikt zwischen familiärer Reproduktion und dem tradierten Verständnis von Wissenschaft als Lebensform (Mittelstrass 1997, S. 15f.), der infolge der Bedeutungszunahme von quantitativen Leistungsindikatoren im wissenschaftlichen Feld (Jungbauer-Gans und Gross 2013; Lutter und Schröder 2016) ein noch größeres Gewicht bekommen hat. Nüchtern konstatiert sie, ,,man gehört der Arbeit [dann] nicht mehr mit Leib und Seele" und verweist damit (implizit) auf die institutionalisierte Erwartung, dass die wissenschaftliche Arbeit „nicht neben dem privaten Leben statt[zufinden hat], sondern in ihm“ (Matthies 2006, S. 157 Herv. i. Orig.), während alles andere der wissenschaftlichen Tätigkeit unterzuordnen ist.

Zugleich weichen diese Fälle von einem typischen Cooling out bei Goffman ab, weil die Diskrepanz zwischen Rollenerwartungen und Rollenerfüllung von den Subjekten selbst erkannt wird und sie sich gleichsam „selbst auskühlen" ${ }^{11}$ und ihre Maßstäbe für beruflichen Erfolg an ihre habituellen Dispositionen anpassen. Gleichwohl lässt sich an dieser Konstellation das befriedende Moment von Cooling out studieren, das Goffman (1952) als dessen wesentliche Funktion hervorhebt. Beide Protagonist*innen lasten die Ursachen für ihre unzureichende Passung in hohem Maße sich selbst an. Eine kritische Reflexion gegenüber den exkludierenden Strukturen des wissenschaftlichen Feldes findet allenfalls unterschwellig statt, etwa wenn Ruprecht erwähnt, wie sehr die Ungewissheit eines beruflichen Erfolgs in der Wissenschaft die Kommunikation unter Kolleg*innen bestimmt. Ob jedoch die Anforderung einer performativen Inwertsetzung von Leistung für den wissenschaftlichen Erkenntnisprozess notwendig oder hilfreich ist - so im Fall Lambrecht - oder ob eine Vergesellschaftungsform angemessen ist, in der berufliche Leistungen (symbolisch wie materiell) eine höhere Wertigkeit haben als Leistungen zur Reproduktion (Becker-Schmidt 2003), wird nicht hinterfragt.

\footnotetext{
11 Vgl. dazu Fürst (2016), der am Beispiel des Umgangs von Autor*innen mit abgelehnten Manuskripten unterschiedliche Strategien des „Selbst-Auskühlens“ herausarbeitet, um das Scheitern erträglicher zu machen.
} 


\subsection{Selbstwirksamkeitsbedürfnis kollidiert mit Strukturen der Arbeitsorganisation - Cooling out als Versuch der Selbstbehauptung}

In der zweiten Konstellation entwickelt sich das Mismatch zwischen Habitusdispositionen und beruflichen Anforderungen aus einem ausgeprägten Drang nach Selbstwirksamkeit ${ }^{12}$ und Selbstbehauptung, der sich an den (hierarchischen) Strukturen und Regeln der Arbeitsorganisationen reibt. Die hier versammelten Fälle haben einen hohen Gestaltungsanspruch und geraten damit immer wieder in das Fadenkreuz organisationaler Restriktionen. Es ist also weniger ein bestimmtes Berufsfeld, an dem sich für die Akteur*innen dieser Konstellation die Nichtpassung festmacht, als vielmehr eine spezifische berufsübergreifende Form der Arbeitsorganisation. Folglich repräsentieren die Fälle ganz unterschiedliche Berufe und haben mitunter auch schon einen oder mehrere Berufswechsel hinter sich.

Mit Blick auf biographische Prägungen fällt auf, dass die Befragten in dieser Mismatch-Konstellation eine konfliktbeladene Kindheit erlebt haben - sei es, dass sich gegen eine strenge und wenig empathische Mutter durchgesetzt werden musste, dass man sich unter einem autoritären Vater zu behaupten hatte, der einem vermittelte, als Mädchen weniger wert zu sein als die männlichen Geschwister, dass man vom Vater verprügelt wurde, während die Mutter schweigend daneben stand, oder dass man von der Mutter zu den Großeltern abgeschoben wurde, weil der neue Lebenspartner nicht mit einem Kind zusammen leben wollte. Derlei Erfahrungen sprechen dafür, dass der Drang nach Selbstbehauptung auf einem bereits frühzeitig ausgebildeten Überwindungsmotiv fußt, wobei die Akteure in ihrem Tun mitunter einen enormen Ehrgeiz entwickeln und zuweilen auch eine ausgeprägte Härte gegen sich selbst an den Tag legen. ${ }^{13}$

Auffällig ist außerdem, dass die Narrative der Interviewten in diesen Fällen einen starken Glauben an die eigenen Fähigkeiten zum Ausdruck bringen. Sie bezeichnen sich als schlau, findig, kompetent, unerschrocken, zupackend oder kommunikationsstark und haben diese Selbstsicht bereits während der Schulzeit ausgebildet. Ein Hochschulmanager berichtet beispielsweise, dass er aufgrund seiner guten Leistungen auf dem Gymnasium von seinen Mitschülern als „Professor“ bezeichnet wurde; ein Politikberater hebt hervor, dass er die Schule einschließlich des Abiturs ,mit geringem Aufwand" geschafft habe. Für einige war die Schule allerdings auch ein konfliktbehafteter Ort, von dem sie sich abgrenzen. Eine Wissenschaftlerin etwa wurde in der siebten Klasse vom Gymnasium ,runter geschmissen“ und kurz darauf auch von der Realschule, weil sie mit den Lehrern „konstant im Krieg“ war eine Attitüde, die sich offensichtlich im Zuge permanenter Gewalterfahrungen mit dem Vater ausgebildet hatte. Sie hat sich stattdessen auf ihre eigene Art und Weise weitergebildet, hat ,sehr sehr früh angefangen zu lesen [...] Philosophie, Politik, Biologie, alles [...] bestimmt pro Woche zwischen fünf und sieben Bücher“.

\footnotetext{
12 In Anlehnung an Bandura (1977) bezeichnet Selbstwirksamkeit hier eine Eigenschaft, Herausforderungen zu suchen, mit denen auf die Umwelt Einfluss genommen und etwas bewirkt werden kann.

13 Hier zeigen sich im übrigen interessante Parallelen zu einer Studie über Erfolgsbiographien (siehe Hänzi und Matthies 2014), in der bei einer Antriebsstruktur der Selbstbehauptung ebenfalls problematische Erfahrungen in der Primärsozialisation identifiziert wurden.
} 
Neben dem starken Gestaltungswillen weisen die Handlungsstrategien dieser Protagonist*innen einen latenten Hang zur Selbstermächtigung auf, der sich auch in bewussten oder unbewussten Regelverstößen äußert. „Wenn ich jemand fragen würde, da würde der sagen, ,das geht nich', also frag' ich nich'“, fasst etwa eine Sozialarbeiterin ihre diesbezügliche Haltung zusammen. Ähnlich beschreibt sich eine Ingenieurin als jemand, die keine Scheu vor der Obrigkeit hat. Mit gewissem Stolz schildert sie im Interview, wie sie mit blau gefärbten Haaren zu einer Betriebsfeier erschienen ist und ihre Vorgesetzten dort mit der Behauptung provozierte, das Unternehmen am Tag zuvor im gleichen Outfit auf einer internationalen Konferenz vertreten zu haben. Im Anschluss an Goffman kann man hier von einer gewissen „Systematizität des Unanständigen“ (Willems 1997, S. 229) sprechen, die womöglich aus einem Vergeltungsdrang gegenüber dem Elternhaus herrührt.

Schon die Berufswahl scheint eher von dem Wunsch geprägt, sich gegen elterliche Bevormundungen aufzulehnen, als dass sie einem ausgeprägten Sachinteresse folgte. Die Entscheidung für ein technisches Studium trotz der damit verbundenen Kürzung des elterlichen Unterhaltsgeldes begründet die Ingenieurin beispielsweise mit dem Bedürfnis, gegen den autoritären Vater zu opponieren, während die konkrete Fächerwahl dem Zufall überlassen wird: „Zwei war'n NC-frei und dann hab' ich ausgezählt".

Ähnlich erscheint das berufliche Feld im Lichte der Selbstbeschreibungen weniger als Ort des geregelten Leistungsaustausches denn als Arena im Kampf um Selbstbehauptung. Konflikte, die infolge des eigenmächtigen „Sich-Raum-nehmens“ und „Relevantsetzens“ entstehen, werden von den Fällen als Limitierung ihrer Handlungsmöglichkeiten und als Missachtung ihres Potenzials gedeutet. Die bereits erwähnte Ingenieurin beschreibt ihr Problem mit dem Arbeitsfeld bspw. so: „Es is’ wirklich so, dass ich manchmal denke, ich renne immerzu gegen an. Ich mache eigentlich die Sachen gut, ich mach' sie anders." Dabei zeigt sich bei den Frauen zuweilen eine Doppelstrategie sowohl einer Abgrenzung gegenüber den männlichen Berufskollegen als auch einer gegenüber der eigenen Genusgruppe, wie die folgende Selbstbeschreibung der Ingenieurin zum Ausdruck bringt: „Ich bin nich' die Ingenieurin, die mitläuft [...], ich war immer 'n bisschen kreativer als die ander'n [...] ich bin auch nich' die typische Fraueningenieurin, weil ich nich' in die Männerrolle schlüpfe, sondern meine Rolle versuche zu behalten“. Niederlagen in diesem Wettstreit werden als Probleme des Berufsfeldes gedeutet, mit solchen Vorzügen umzugehen, etwa ,weil Männer Sündenböcke suchen“ oder weil die Institutionen die besonderen Kompetenzen der hier betrachteten Protagonist*innen nicht zu schätzen wissen.

Für die Fälle dieser Cooling-out-Konstellation gehören Mismatch-Erfahrungen gewissermaßen zum Berufsalltag. Folglich blicken die hier versammelten Akteur*innen auf wiederholte, gleichsam wellenförmig auftretende Cooling-outProzesse zurück, die allerdings kaum zu einer Änderung des Selbstbildes führen. Auf eine Phase der Enttäuschung und Motivationsabnahme, die zumeist infolge eines spontanen Ereignisses oder bereits länger gärenden Konflikts eintritt, folgt in der Regel ein „try again“ (Wei 2016) dergestalt, dass die Akteur*innen sich einen anderen Arbeitsplatz oder ein neues Tätigkeitsfeld suchen, von dem sie sich mehr Freiraum für ihre berufliche Entfaltung versprechen. 
Am Beispiel der Werdegänge der Sozialarbeiterin Barbara Henckes und des ITBeraters Steffen Ammermann soll dieser wellenförmige Verlauf nun expliziert werden. Beide Protagonist*innen wachsen in einem von Emotionslosigkeit und Pflichterfüllung geprägten Elternhaus auf, wenngleich ihre Ausstattung mit kulturellem Kapital sich erheblich unterscheidet: Henckes Eltern sind beide Ärzt*innen, Ammermanns Vater ist ungelernter Stahlarbeiter und die Mutter Hauswirtschaftsgehilfin.

Henckes Drang nach Selbstwirksamkeit bildet sich schon frühzeitig aus. Zum einen leidet sie unter der Strenge und moralisierenden Einengung der stark religiös orientierten Mutter und entwickelt dagegen Auflehnungsstrategien, zum anderen muss sie sich als einziges Mädchen gegen vier Brüder durchsetzen. Mit einer „Rasselbande" anderer Kinder zieht sie durch die Straßen und treibt allerlei Unfug, weshalb sie aus erzieherischen Gründen von ihren Eltern auf eine katholische Mädchenschule geschickt wird, die sie als „Horror“ erlebt. Gegen den Willen der Mutter bricht sie mit Sechzehn die Schule ab und beginnt eine Lehre als Buchhändlerin. Eine Affinität zu Büchern hat sie durch ihren Großvater entwickelt, der ein „DichterFreak" war, aber auch durch die Mutter, die sie auch mal zu schwieriger Lektüre animiert hat. Hinzu kommen bei der Entscheidung pragmatische Erwägungen: ,Weil ick schon immer Nachteule war und lange jeschlafen hab" ", sagt sie, kam nur ein Beruf in Frage, bei dem die Arbeit erst später am Morgen beginnt.

Nach Abschluss der Lehre geht Henckes als Au-pair nach Paris, um endlich der Enge des elterlichen Zuhauses zu entkommen. Dort gerät sie jedoch an eine „grauenhafte Familie“, wird ,,sofort krank“ und muss wieder zurück nach Hause, wo sie ihre Optionen abwägt: „Soll ich im Buchhandel arbeiten? Nee. Also studier’n!“ — eine Schlussfolgerung, die angesichts des nicht vorhandenen Abiturs überrascht, vor dem Hintergrund des akademischen Elternhauses jedoch einen gewissen Sinn ergibt. Scheinbar streng rational klopft sie die objektiven Möglichkeiten und Konsequenzen ab und kommt am Ende zu dem Ergebnis: „Na ja, natürlich Sozialarbeit!“ Die Entscheidung trifft sie, weil man da ,wenig tun muss“.

Als Ausbildungsstätte wählt Henckes eine katholische Institution und begründet das damit, dass sie denen aufgrund ihrer schulischen Erlebnisse noch was heimzahlen wollte. „Und so war's auch. [...] Gleich Rote Zelle“, beschreibt sie triumphierend ihren Studienbeginn. Für das Studium bleibt angesichts des politischen Aufbegehrens nicht viel Zeit, die erforderlichen Leistungen schüttelt sie sich mit Minimalaufwand ,,aus'm Ärmel“", sodass sie erst im Berufspraktikum ,richtig gelernt (hat), was Sozialarbeit is" ".

Zunächst macht der neue Beruf „richtig Spaß“. Die erste Stelle in einer Kindertagesstätte bietet Gestaltungsmöglichkeiten und wird von Henckes mit den Worten bewertet: „Ich konnte sozusagen mich da ein bisschen entfalten“. Doch mit der Zeit stößt sie dabei auch an Grenzen und nach etwa eineinhalb Jahren stellt sich das Gefühl ein: „reichte“. Sie wechselt in die Elternarbeit und findet dort die offene Beratung ,herrlich“ - bis eine neue Amtsleitung eingesetzt wird und auf das Einhalten von behördlichen Vorgaben wie etwa Vergaberichtlinien pocht, ,,allet mit drei Kostenvoranschlägen“, was Henckes als beengend empfindet und den Gedanken aufkommen lässt: „Ach eig'ntlich könnte ma' wieder was anderes komm'n“. Nach vier Jahren Tätigkeit auf dieser Stelle kündigt sie und lässt sich von einem ehemaligen Kollegen für einen neu eröffneten Buchladen anheuern, ,natürlich links- 
liberal". Dort langweilt sie sich aber schon bald und als irgendwann auch noch die Geschäfte schlechter gehen, besinnt sie sich darauf, dass sie ja noch einen zweiten Beruf hat. Sie bewirbt sich für die stellvertretende Leitung einer heilpädagogischen Einrichtung und setzt sich dort ,gegen ziemlich viele Bewerbungen durch“. Das gibt ihr neuen Elan und lässt sie sogar in Kauf nehmen, verbeamtet zu werden, was sie eigentlich nie wollte. Allerdings ist das Verhältnis zu den Vorgesetzten auch hier konfliktbehaftet, die Heimleiterin ,schwierig“. Vor allem missfällt es Henckes, dass sie sich „unterordnen muss“. Viele Jahre kompensiert sie ihren Unmut durch die Arbeit mit den Jugendlichen und diverse Weiterbildungen, die sie als regelrechten Qualifizierungsschub erlebt und die ihr das Gefühl der fachlichen Passung vermitteln: „Da ha' ick geseh'n, kann ick allet“. Irgendwann aber empfindet sie die Arbeit als „wieder so kleinkariert“ und sich selbst in ihren Möglichkeiten limitiert. „Ich war ein bisschen reduziert", fasst sie ihr Befinden zusammen. Nach acht Jahren lässt sie sich schließlich von einer Freundin überreden, zu ihr an eine Fachhochschule zu kommen und dort die Fort- und Weiterbildung zu organisieren. Vor dem Hintergrund ihrer bisherigen Erfahrungen der Restriktion und eingeschränkten Handlungsfähigkeit entscheidet Henckes allerdings: „Ick mach nur mit halber Stelle“. Die andere Hälfte will sie freiberuflich arbeiten. Finanziell schien ihr das machbar, denn die Arbeit war nicht schlecht dotiert und ihre Lebenshaltungskosten nicht so hoch. Aber auf der Stelle selber geht es ihr diesmal ,richtich dreckich“. Mehr noch als bisher sieht sie sich hier in ihren Handlungsmöglichkeiten eingeengt. Als besonders schmerzvoll erlebt sie den Umgang mit einem von ihr entwickelten Vorschlag zum institutionellen Ausbau der Weiterbildung. Nicht nur, dass sich dessen Bearbeitung in die Länge zieht, am Ende muss sie sich dafür auch noch ,abkanzeln lassen“. Daraufhin hat sie „,kein’ Bock, da hin zu geh'n“ und macht erstmals in ihrem Leben auf einer Arbeitsstelle ,blau“. Nachdem sie die Arbeit wieder aufgenommen hat, schwankt sie zwischen Auflehnung und Rückzug, Frechheit und Abgestumpftheit, was sie aber zunehmend ermüdet. „Det war so anstrengend, dat Frechsein“, resümiert sie. Hinzu kommt, dass sie aufgrund ihres Beamtenstatus die freiberufliche Nebentätigkeit nicht im gewünschten Umfang ausüben darf, worüber sie sich ebenfalls empört: „Da hab' ich diese Fessel so richtich gemerkt, dass mich da der Status hindert, det zu machen, wat ick will, ja. Frechheit““. In Anbetracht dieses sie lähmenden Zustands kommt sie nach acht Jahren zu dem Schluss: „Du musst kündigen!“ Sie sucht aktiv nach anderen Optionen und lässt sich schließlich, nunmehr 51-jährig, auf Anraten eines Coaches ,aus arbeitsmarktpolitischen Gründen“ beurlauben. Über das Angebot eines Berufskollegen erschließt sie sich das Feld der Supervision und beginnt, in einer gemeinsamen Praxis mit ihm freiberuflich als Beraterin und Coachin tätig zu sein - ein Schritt, den sie als befreiend beschreibt.

Steffen Ammermanns Selbstwirksamkeits- und Selbstbehauptungsdrang lässt sich zum einen ebenfalls auf das Bedürfnis zurückführen, das als hart und deprimierend erlebte Herkunftsmilieu zu überwinden, zum anderen aber auch auf einen dort ausgebildeten Klassenethos und Prinzipien der Solidarität und Fairness, die er sich bewahren möchte. Sieht er diesbezüglich Verletzungen, nimmt er eine widerständige Haltung ein und tritt, in Umkehrung eines berühmten Zitats ${ }^{14}$, ,nach oben“ oder

14 Heinrich Mann: Der Untertan. 
- in seinen Worten - sagt, „,dass das Scheiße is'“. Dadurch kommt es immer wieder zu Konflikten mit Vorgesetzen: „kommt nich’ gut an“, wie er etwas untertreibend resümiert.

Ammermann wächst in einer Kleinstadt auf, ,so 'ne Arbeitersiedlung“, wo er und sein zwei Jahre älterer Bruder ,die einzigen [war'n], die da studiert haben“. Aber erst einmal besucht er die örtliche Schule und lernt im Anschluss an den Hauptschulabschluss ,'n Handwerk“, im Stahlwerk, wo schon Vater und Großvater gearbeitet haben. Während der Ausbildung wird er zum Jugendvertreter gewählt und setzt sich für gute Arbeitsbedingungen ein. Nach dreieinhalb Jahren schließt er die Ausbildung als Energieanlagenelektroniker ab und wird vom Betrieb übernommen, aber aufgrund der damaligen Stahlkrise „nich' im Beruf, also direkt an'n Hochofen dann“, wo Teamgeist, Zusammenhalt, Vertrauen und Solidarität gewissermaßen Überlebensprinzip sind. „Also wenn du da 'n Fehler gemacht hast“, so Ammermann, ,haben alle drunter gelitten“.

Das Stahlwerk ist in Ammermanns Narrativ die einzige berufliche Station, in der er zwischenmenschliche Kontakte als vollumfänglich positiv beschreibt, die Vorgesetzten eingeschlossen. Es ist der Ort, der ihn stark geprägt hat und der als Referenzfolie für sein späteres berufliches Leben dient. Dennoch will er dort weg. Denn das Milieu hat auch Schattenseiten, ,diese [Schichtarbeit], Dreck und alles“. Bekräftigt wird sein Drang zum Milieuwechsel durch seinen Vorgesetzten, der ihm rät, etwas aus seinem Leben zu machen und ihm auch das intellektuelle Potenzial dazu attestiert: „Der war 'n Türke und der sachte, ,Junge, sieh zu, mach irgendwas, du bist doch nich' doof, lern was, geh hier weg'.“

Erst einmal geht Ammermann zur Bundeswehr. Eigentlich wollte er verweigern, aber er fürchtet, dass das eine längere rechtliche Auseinandersetzung nach sich ziehen und die Suche nach einem anderen Arbeitsplatz erschweren würde. Am letzten Tag des 18-monatigen Wehrdienstes verweigert er dann doch, damit er später nicht zu Reserveübungen eingezogen wird. Beruflich landet er danach wieder im Stahlwerk, weil die Bewerbungen als Elektriker nicht geklappt haben, „natürlich nich”“, sagt er, als wäre das ein vorgezeichnetes Schicksal gewesen. Doch sein Wunsch, vom Stahlwerk wegzukommen, besteht immer noch, zumal die Schattenseiten dort noch deutlichere Konturen angenommen haben. Einige Kollegen erlebt er nun als so „fertig“, dass sie schon am Morgen zur Schnapsflasche greifen und „denn da durch die Halle getorkelt sind, wo wir manchmal Angst hatten, dass die in 'n Schacht fall' $n$ “. Und als er sich eines Tages dabei ertappt, in seinem Privatleben Verhaltensweisen an den Tag zu legen, wie er sie von seinem Vater kennt, sagt er sich, ,so, jetz' musste hier aber sofort raus“. Auslöser war, dass er seine Freundin angeschrien hat, weil sie ihn nach einer Nachtschicht versehentlich geweckt hatte. „Das war denn wie so 'n Filmriss, wo ich dachte, halt, diese Situation kennste doch, ja, das war das, was ich früher von mei'm Vadder erlebt hab' [...] und das war so denn der Punkt, wo ich gesacht hab', irgendwie raus. [...] ich bin jetz' zwanzich und so, das kann nich' die Welt sein“.

Über Bekannte erfährt Ammermann von der Möglichkeit, an einer bestimmten Hochschule über ein Aufnahmeverfahren zum Studium zugelassen zu werden. Diese Option ergreift er, sagt sich, ,einfach ma' probier’n“, und bereitet sich gründlich auf die Prüfung vor. Mit Erfolg: Im Jahr darauf kann er das Studium beginnen. Zur 
Finanzierung bekommt er sogar ein Stipendium von einer gewerkschaftsnahen Stiftung, das ihm allerdings nach drei Semestern „,wegen Abweichlertum“ gestrichen wird. Diese Disziplinierungsmaßnahme veranlasst ihn nicht zur Anpassung, sondern bestärkt ihn eher in seiner Haltung: „Dann hab' ich gesacht, ihr könnt mich mal, ich bin Elektriker“. Er greift auf seine handwerkliche Kompetenz zurück und verdient sich seinen Lebensunterhalt mit Altbausanierungen, die er neben dem Studium durchführt.

Das Studium selber „war gleich super. [...] das war gut investiertes Geld“, sagt Ammermann mit Blick auf die Kosten, die er dafür auf sich genommen hat. Er spricht damit vor allem die soziale Dimension an, die sich für ihn als gar nicht so fremd entpuppte, wie er befürchtet hatte. Denn an der Universität trifft er auf seinesgleichen, Leute, die ebenfalls schon im Berufsleben gestanden haben. Das Studienfach - als Hauptfach wählt er Soziologie - scheint demgegenüber zweitrangig zu sein; in der Selbstbeschreibung seines Werdegangs findet es kaum weitere Erwähnung. Auch dass er sich nach Abschluss des sechssemestrigen Studiengangs am gleichen Ort an einer Universität für die Fortsetzung des Soziologiestudiums einschreibt, hat eher pragmatische Gründe, nämlich ,zwei Auslandssemester“ zu machen, wie er es nennt, und in das Mutterland der Eltern seiner Frau zu reisen. Wieder zurück wechselt er in die Sportwissenschaft, worauf ihn ein Bekannter gebracht hat, der das auch machen wollte, um hinterher in der Gesundheitsprävention zu arbeiten. „Das war damals grade so 'n Trend“", sagt Ammermann. Seinen Lebensunterhalt verdient er sich die ersten zwei Jahre als Sozialarbeiter in einem Jugendheim und im letzten Studienjahr als Manager eines Sportvereins. Nach sechs Semestern schließt er, inzwischen 30-jährig, das sportwissenschaftliche Studium mit dem Diplom ab. Thema seiner Diplomarbeit sind die gesundheitlichen Auswirkungen der Schichtarbeit, womit er noch einmal seinen beruflichen Wurzeln im Stahlwerk huldigt.

Als nunmehr diplomierter Sportwissenschaftler findet Ammermann bei einer Krankenkasse eine Stelle als Fachbereichsleiter für gesundheitliche Prävention. Zu seinen Aufgaben gehören Projekte wie ,gesund alt werden“, in die er mit viel Engagement und großem Gestaltungsanspruch einsteigt. Doch schon bald empfindet er sich vom Gros der Mitarbeiter*innen ausgebremst. Das waren Sozialfachangestellte, sogenannte „Sofas“, berichtet Ammermann, ,und wie der Name sacht, so verhalten die Leute sich auch“. Vorschläge, die er zusammen mit anderen Akademikern aus dem Kolleg*innenkreis zur Verbesserung von Arbeitsabläufen unterbreitet, stoßen auf wenig Gegenliebe. Als der Arbeitsbereich infolge der Gesundheitsreform aufgelöst wird und Ammermann ins Marketing versetzt wird, hat er folglich zunächst auch kein Problem damit. „Also Marketing war schon interessant [...] man hätte was draus machen können“, sagt er. Doch macht ihm nun sein neuer Vorgesetzter und dessen Umgang mit der Belegschaft zu schaffen: „Das war'n Choleriker“. Im Kontrast dazu beschreibt Ammermann sich als Mann mit Rückgrat, der als einziger intervenierte (,keiner traute sich was zu sagen“), wenn der Chef seinen „herabwürdigend[en]“ Umgang an den Tag legte. Irgendwann „,eskalierte“ der Konflikt, Ammermann bekommt Telefonverbot und seinen Kolleg*innen wird untersagt, mit ihm zu reden. Über einen Rechtsanwalt setzt er durch, bei Fortzahlung seiner Bezüge freigestellt zu werden, bis die vom Arbeitgeber veranlasste Kündigung rechtswirksam wird eine Lösung, die Ammermann im Rückblick als trickreich und als Erfolg rahmt. 
Dem Beschäftigungsverhältnis trauert er nicht nach, weil das ,extrem Feudalherrschaft [war] [...] also ich hatte da nie 'ne reelle Chance, dass ich da hätte überleben könn'n“".

Veranlasst und finanziert durch das Arbeitsamt macht er unter dem Titel „Akademikerpotentiale sichern“ nun eine Fortbildung zum Projektmanager in der ITBranche. Im Gegensatz zur Gesundheitsbranche erhofft er sich hier bessere Zukunftschancen. Zunächst arbeitet er in einer kleinen Firma, wo er schon während der Fortbildung ein Praktikum gemacht hat. Dort fühlt er sich wohl, weil er weitgehend frei Dinge in Bewegung setzen kann: „Da war ich Chef“, beschreibt er seine dortige Stellung, und als sich die Eigentümer nach drei Jahren zerstreiten und das Unternehmen auflösen, versucht Ammermann, das Unternehmenskonzept in etwas abgewandelter Form in Eigenregie fortzuführen. Nach zwei Jahren muss er allerdings feststellen: „Es rechnet sich nicht“. Er beginnt eine neue Angestelltentätigkeit in einem IT-Unternehmen, diesmal als ,,Vertriebler“, was etliche Jahre auch gut läuft. Es gelingt ihm, ein Verkaufsgebiet aufzubauen, das sich sehen lassen kann, was er auf seine kommunikativen Fähigkeiten und seine redliche Art im Umgang mit den Kunden zurückführt. In der Beschreibung charismatisiert er sich als „Telefonsau“, als jemand, „,der sehr stark im Telefonieren“ ist und dennoch keine unlauteren Geschäftsmethoden anwendet: „Ich hab' die Leute nich' über'n Tisch gezogen“. Dann vergrößert sich die Firma durch einen Unternehmensaufkauf, es wird umstrukturiert und von Ammermann werden Verkaufszahlen erwartet, die er für unrealistisch hält. Zudem empfindet er die strengen Vorgaben als Abwertung seiner bisherigen Leistung und als Vertrauensdefizit ihm gegenüber. Das verletzt seinen Stolz und er kündigt. Ein anschließendes Angebot der Geschäftsführung, mehr Freiraum zu bekommen, schlägt er aus; eine weitere Zusammenarbeit ist für ihn aufgrund der erfahrenen Abqualifizierung seiner Leistung undenkbar.

Doch Ammermanns Versuch der Selbstbehauptung läuft gewissermaßen ins Leere. Auch beim nächsten Arbeitgeber wird er mit Verkaufserwartungen konfrontiert, die aus seiner Sicht nur mittels unlauterer Praktiken zu erfüllen wären. Anstatt sich erneut aufzulehnen, reagiert er dieses Mal allerdings mit einer stillen Verweigerungshaltung: Er macht sein Hobby zum Beruf und klappert, teils auch während der Arbeit, Flohmärkte ab. Seine Verkaufszahlen gehen in der Folge runter statt rauf und Ammermann wird zu einem Gespräch bei der Geschäftsleitung zitiert, dessen Verlauf er folgendermaßen wiedergibt: ,....und dann eskalierte das so jetz', dass die Leute gesacht hab'n, ,Sie sind gar nich' mehr so bei der Sache und irgendwie so die Erfolge, wir wissen nich', ob sie nächstes Jahr noch erfolgreich sein werden. Mein' ich, ,jo, ich werde nich' erfolgreich sein', ja und ,denn müssen wir uns trennen. Tschüss!‘, ja und denn wurd' ich dem freien Markt wieder übergeben“. Ein Einlenken zog Ammermann auch dieses Mal nicht in Betracht, das hätte er als Demütigung empfunden, was seiner Grundhaltung zutiefst widerspricht. Und um noch einmal zu unterstreichen, wie sehr er mit seiner Haltung im Recht war, fügt er hinzu: „Weil ich weiß, ich werde dann nich' mehr erfolgreich sein, auch wenn ich hundert Prozent oder noch mehr geben würde, der Markt gibt's einfach nich' mehr her". So wird Ammermann im Rahmen der gesetzlichen Kündigungsfrist entlassen und bis dahin mit sofortiger Wirkung freigestellt. Binnen weniger Wochen findet er eine neue Beschäftigung, diesmal als Integrationshelfer in einer Sammelunterkunft für 
Flüchtlinge. Hier hofft er, mit seinen moralischen Ansprüchen besser aufgehoben zu sein, und gefällt sich dabei zugleich in der Pose des Aufmüpfigen: „Das is' doch durch die Presse gegang'n mit diesen 6000 neuen Wohnungen“, berichtet er, „da steh'n doch die ganzen Anwohner auf den Barrikaden [...] und wir können da als Sozialarbeiter arbeiten - das wird schön“.

Dieses habituelle Element der Widerständigkeit im Drang nach Selbstwirksamkeit ist gleichsam idealtypisch für die Fälle dieser Konstellation. So erscheinen die beruflichen Werdegänge in der Gesamtschau als ein ewiger Kampf gegen die Widrigkeiten des Erwerbssystems - gespeist aus dem Anspruch, ,,wirksam in der Arbeit zu sein" (Hardering 2017, S. 47), die Maßstäbe dafür aber, so gut es geht, selbst zu setzen, um ein ,aufgeklärter Mensch“ zu bleiben und kein „Knecht des Kapitals“ zu werden, wie etwa Ammermann sein Credo beschreibt. Man könnte auch sagen, der in der Primärsozialisation erworbene Habitus dieser Akteur*innen ist derart robust, dass die berufliche Sozialisation hier permanent gegen die innere Akzeptanz anläuft. Entsprechend werden die erfahrenen beruflichen Probleme von den Subjekten nicht auf persönliche Defizite zurückgeführt, sondern dem Berufsfeld angelastet, das das Potenzial solcher Akteur*innen nicht zu nutzen weiß. „Ich war ein bisschen reduziert", nennt das die ehemalige Sozialarbeiterin Henckes. Ausdruck dieser Deutung ist nicht zuletzt, dass etliche Interviewte ihren anschließenden beruflichen Weg als Triumph rahmen, getreu dem Motto: „Seht her, zu was ich fähig bin und was ihr Euch habt entgehen lassen". Selbst wenn der berufliche Wechsel mit einem Statusverlust oder finanziellen Einbußen verbunden ist, erschüttert das Cooling out nicht ernsthaft den Glauben an den Selbstwert und die eigene Kompetenz. Was hier sukzessive ,auskühlt“ ist vielmehr die Illusio, der Glaube, den Anspruch auf ein „Selbstsein“ im Beruf unter den gegebenen Verhältnissen in den gewöhnlichen Institutionen des Berufsfeldes einlösen zu können.

Auch in dieser Konstellation gestaltet sich der Cooling-out-Prozess weitgehend in Form des „Selbst-Auskühlens“, bei dem die Fälle sich gleichsam im Sinne einer inneren Kündigung von ihrem Commitment gegenüber der Organisation verabschieden. Dabei zeigen sich deutliche Parallelen zu Strategien des „Excusing“ und „Refusing", die Fürst (2016) bei Autor*innen im Umgang mit abgelehnten Manuskripten herausgearbeitet hat. „An excuse happens“, so Fürst (ebd., S. 163), „when the writer has accepted the occurrence of failure but does not claim full responsibility for having failed", etwa weil die Bewertungskriterien unklar oder sogar falsch waren - eine Strategie, zu der sich vor allem im Fall Ammermann Parallelen finden. Im Unterschied dazu bezeichnet „Refusing“ das Verhalten einer Person, ,, who dismisses the failure and denies responsibility“ (ebd., S. 168) - eine Strategie, die u.a. stark auf den Fall Henckes zutrifft.

\section{Fazit und Ausblick}

Mit dem vorliegenden Beitrag wurde das Ziel verfolgt, die handlungsleitende Grammatik von Berufswechseln aus einer Cooling-out-Perspektive zu rekonstruieren. Dabei wurde Goffmans Cooling-out-Konzept um eine habitustheoretische Perspektive erweitert und Cooling out als Folge eines Mismatch von Habitus und Feld kon- 
zeptualisiert. Vorgestellt wurden zwei Mismatch-Konstellationen mit je spezifischen Diskrepanzen zwischen habituellen Dispositionen und Felderwartungen. In der ersten Konstellation entzündet sich das Cooling out an einer unzureichenden Übereinstimmung von Karriereaspirationen, Risikobereitschaft und subjektiv empfundenen Karrierechancen. Die Fälle dieser Konstellation starten ihren Berufsweg typischerweise mit klaren Karrierevorstellungen, geraten aber im Laufe ihrer beruflichen Entwicklung in Zweifel, ob sie ihre ambitionierten Ziele vor dem Hintergrund der im Berufsfeld wahrgenommenen Bedingungen auch erreichen werden. In der zweiten Konstellation wird das Cooling out durch eine Diskrepanz zwischen Selbstwirksamkeitsansprüchen und Selbstbehauptungsgebaren seitens der Subjekte und wahrgenommenen Entfaltungsmöglichkeiten im Korsett organisationaler Regeln ausgelöst.

Mit Blick auf den prozessualen Charakter von Cooling out ergibt sich hingegen ein relativ übereinstimmendes Bild. Beide Konstellationen weisen eine typische Abfolge von Phasen auf, wie sie bereits in der Literatur beschrieben wird (Thomas 2014; Wei 2016): Nach dem Erleben von Verunsicherungen oder Enttäuschungen kommt es zum Erkennen von Diskrepanzen zwischen den eigenen beruflichen Ansprüchen und den Erwartungen des beruflichen Feldes und infolgedessen zum Überdenken sowie gegebenenfalls Modifizieren der eigenen Ansprüche und zur Suche nach alternativen beruflichen Optionen. Zuweilen ist diese letzte Phase bereits mit einem sukzessiven Erwärmen für die gefundene Alternative verbunden. Dabei zeigen sich Parallelen zu zweien der bei Hardering und Will-Zocholl (2019) herausgearbeiteten „Modi der Aneignung von Arbeit“. So finden wir bei den Fällen der ersten Konstellation, bei denen die ursprünglich ambitionierten beruflichen Ziele im Zuge des Cooling out auf ein eher auf Pflichterfüllung orientiertes Verhältnis zur Arbeit heruntergedimmt werden, ebenfalls einen eher pragmatischen Modus der Sinnbewahrung und in der zweiten Konstellation deutlich widerständige Formen. Anders als Hardering und Will-Zocholl in ihrer Untersuchung herausgefunden haben (vgl. ebd., S. 285, $288 \mathrm{ff}$.) rekurrieren die Fälle dieser Konstellation allerdings nicht auf eine spezifische Berufsethik, sondern auf ihr individuelles Wertesystem und geraten dabei immer wieder in Konflikt mit den Arbeitsorganisationen. Entsprechend blicken sie auch auf wiederholte Cooling-out-Erfahrungen zurück, deren biographische Tragweite von Mal zu Mal zunimmt, während das Cooling out für die Fälle der ersten Konstellation ein zwar einschneidendes, aber zugleich einmaliges berufsbiographisches Ereignis zu sein scheint.

Die Divergenzen zwischen den Mismatch-Konstellationen gehen mit unterschiedlichen Effekten von Cooling out einher. In der ersten Konstellation schreiben die Subjekte die Gründe für den Berufswechsel sich selbst zu und führen ihn darauf zurück, ein falsches berufliches Feld ausgewählt zu haben. Sie entwickeln eine Selbstverschuldungsüberzeugung und stellen folglich die Regeln und Praktiken der Institutionen, in deren Kontext sie sich zu ihrer Entscheidung genötigt sahen, nicht infrage. In dieser Konstellation wird mit dem Prozess des „Auskühlens“ also vermieden, dass die ,Verlierer“ auf destruktive Weise, etwa durch deviantes Verhalten, auf ihre Niederlage reagieren. Ganz im Sinne Goffmans hat Cooling out hier sowohl eine exkludierende (Aspekt der Statusdegradierung) als auch eine inkludierende (Vermittlung eines neuen passfähigeren Selbstbildes) Funktion. Anders in der zweiten Konstellation: Hier wird der Berufswechsel nicht als Statusdegradierung aufgefasst, 
sondern mit der Hoffnung auf einen Statusgewinn im Sinne von größerer Autonomie verbunden. Folglich entwickeln die Subjekte in dieser Konstellation auch keine Selbstverschuldungsüberzeugung, sondern richten die Schuldzuschreibung für ihren beruflichen Wechsel an das Feld bzw. die dazu gehörenden Institutionen und Personen, durch die sie ihre Identität bedroht sehen. Mit dieser Sichtweise rechtfertigen sie auch deviantes Verhalten (z.B. „blau machen“ oder private Tätigkeiten während der Arbeitszeit). Aus funktionalistischer Perspektive könnte man hier deshalb von einem misslungenen Cooling out sprechen. Die Mismatch-Erfahrungen haben zwar einen exkludierenden Effekt, doch die inkludierende Wirkung ist begrenzt, da die Subjekte im Zuge ihrer wiederholten Cooling-out-Erlebnisse mehr und mehr den Glauben verlieren, ihren Wunsch nach „Selbstsein-dürfen“ im Beruf unter den gegebenen gesellschaftlichen Verhältnissen realisieren zu können. Gleichwohl hat das Cooling out für die Subjekte auch in dieser Konstellation eine Selbstschutzfunktion (Goffman 1952, S. 452), indem es einen Fluchtpunkt, gleichsam ein therapeutisches Angebot darbietet, das ein Versöhnen mit dem wiederholten beruflichen Scheitern ermöglicht.

Für beide Konstellationen ist bemerkenswert, dass die Mismatch-Erfahrungen der Subjekte mit Veränderungen in den Berufsfeldern zusammentreffen. Sie lassen sich zum einen mit den Begriffen „Subjektivierung“ und „Entgrenzung“ von Arbeit umreißen, mit denen das seit einigen Jahrzehnten beobachtbare Phänomen eines erweiterten Zugriffs auf menschliche Handlungspotenziale und deren ökonomische Vernutzung thematisiert wird (Kleemann und Voß 2010, S. $434 \mathrm{ff}$.), das in unserem Zusammenhang neben einer allgemeinen Extensivierung der Leistungserwartung vor allem in Gestalt von Verantwortungsverlagerung auf die Subjekte, Zunahme des Wettbewerbs zwischen den Beschäftigten und der Bedeutungszunahme von interaktiver Arbeit und Emotionalität (vgl. Penz und Sauer 2016) zum Tragen kommt. Zum anderen betrifft es die gleichsam sich kontradiktorisch dazu verhaltende Tendenz zur „Bürokratisierung“ (Mayntz 1980), die den Subjekten in Form von autoritärer Führung und widersinnig erscheinenden formalen Regeln aufstößt. An diese neuen Anforderungen können sich die Betroffenen nicht anpassen, weil sie ihnen habituell nicht entsprechen oder ihren Vorstellungen zur Gestaltung von guter und sinnvoller Arbeit zuwiderlaufen.

Insofern können die hier vorgestellten Mismatch-Konstellationen auch als Indiz dafür gelesen werden, dass die im Kontext von strukturellen Veränderungen erwünschten habituellen Anpassungen nicht immer gelingen (Bourdieu 1997, S. $202 \mathrm{ff}$.) und folglich misslungene „Umprogrammierungen“ des Habitus stets mitgedacht werden müssen. Schon Bourdieu hat in diesem Zusammenhang mehrfach auf die Trägheit des Habitus hingewiesen und dies auf seine Genese zurückgeführt: „Über den Habitus regiert die Struktur, die ihn erzeugt hat, die Praxis und zwar nicht in den Gleisen eines mechanischen Determinismus, sondern über die Einschränkungen und Grenzen, die seinen Erfindungen von vornherein gesetzt sind." (Bourdieu 1993, S. 102f.) Der Strukturierung einer schulischen Erfahrung liegt beispielsweise der innerhalb der Familie erworbene Habitus zugrunde, der Strukturierung beruflicher Erfahrung die Erfahrungen aus Schule und Kindheit usw. (Bourdieu 1976, S. 188f.). Diese frühen Phasen der Sozialisation prägen folglich die Grundformation des Habitus, mit der ein Subjekt in die Welt des Berufs eintritt und die sich 
- wie die hier präsentierten Fälle auf eindrucksvolle Weise belegen - als relativ veränderungsresistent erweist.

Mit der Einnahme der habitustheoretischen Perspektive bei der Rekonstruktion von Berufswechseln konnte aber auch herausgearbeitet werden, dass die Institutionen des Erwerbssystems über Mechanismen verfügen, die jenen Akteur*innen, die nicht hinreichend auf die (neuen) Anforderungen des Feldes (um-)programmierbar sind, stillschweigend vermitteln, dass sie hier (mittlerweile) fehl am Platze sind. Es ist also ein „Zusammenspiel von Fremd- und Selbsttypisierung“ (Solga 2004, S. 113), über das die Konstruktion von ,unpassend“ erfolgt. Das spricht dafür, den Cooling-out-Begriff dahingehend zu erweitern, dass er auch die subjektive Verarbeitung von ,institutionalisierten Reaktionen“ (Nadai 2007, S. 446) auf habituelle Nichtpassungen oder misslungene Umprogrammierungen eines individuellen „Primärhabitus“ (Lettke 1996, S. 44 ff.) auf die feldspezifische Rolle einschließt. Beobachtbare Cooling-out-Prozesse wären demnach nicht nur als Ergebnis gewollter Selektionen einzuordnen, sondern auch als Folge misslungener Integration in Betracht zu ziehen.

Offen bleibt die Frage nach der Generalisierbarkeit der Befunde. Obwohl die der Untersuchung zugrunde liegenden Fälle sich durch verschiedenartige Ansprüche an das Berufsleben auszeichnen (z.B. Erfolg vs. Selbstwirksamkeit), repräsentieren sie vornehmlich jenen Typus von Erwerbstätigen, für die Arbeit und Beruf ein wichtiges „Element eines gelingenden Lebensvollzugs“ (Honneth 2008, S. 330) darstellen. Es fehlen diejenigen, die Erwerbsarbeit nur als fremdbestimmt erleben und Sinn in Aktivitäten außerhalb der Erwerbsarbeit suchen. Diese Begrenzung mag nicht zuletzt dem Umstand geschuldet sein, dass sich bei der Akquise von Interviewpartner*innen nur solche Fälle angesprochen fühlten, denen es wichtig war, sich mitzuteilen, während jene, die eher zu Zurückhaltung neigen oder die ein Cooling out gar nicht als solches wahrgenommen haben, über diesen Weg nicht erreicht werden konnten. Dieses Spektrum von Erwerbstätigen in den Blick zu nehmen, bleibt ein Desiderat für künftige Studien.

Danksagung Besonderer Dank gilt Stella Rehbein, die nicht nur die diesem Beitrag zugrunde liegende empirische Arbeit wesentlich mitgetragen, sondern auch wichtige theoretische Anregungen in das Projekt und damit auch in diesen Beitrag eingebracht hat. Bedanken möchte ich mich außerdem bei den Mitautor*innen dieses Schwerpunktheftes sowie bei den anonymen Gutachter*innen und den Herausgeber*innen für ihre konstruktiven Hinweise zur Schärfung des Arguments.

Funding Open Access funding enabled and organized by Projekt DEAL.

Open Access Dieser Artikel wird unter der Creative Commons Namensnennung 4.0 International Lizenz veröffentlicht, welche die Nutzung, Vervielfältigung, Bearbeitung, Verbreitung und Wiedergabe in jeglichem Medium und Format erlaubt, sofern Sie den/die ursprünglichen Autor(en) und die Quelle ordnungsgemäß nennen, einen Link zur Creative Commons Lizenz beifügen und angeben, ob Änderungen vorgenommen wurden.

Die in diesem Artikel enthaltenen Bilder und sonstiges Drittmaterial unterliegen ebenfalls der genannten Creative Commons Lizenz, sofern sich aus der Abbildungslegende nichts anderes ergibt. Sofern das betreffende Material nicht unter der genannten Creative Commons Lizenz steht und die betreffende Handlung nicht nach gesetzlichen Vorschriften erlaubt ist, ist für die oben aufgeführten Weiterverwendungen des Materials die Einwilligung des jeweiligen Rechteinhabers einzuholen.

Weitere Details zur Lizenz entnehmen Sie bitte der Lizenzinformation auf http://creativecommons.org/ licenses/by/4.0/deed.de. 


\section{Literatur}

Allmendinger, J., Stebut, J. von, Fuchs, S., \& Brückner, H. (1999). Eine Liga für sich? Berufliche Werdegänge von Wissenschaftlerinnen der Max-Planck-Gesellschaft. In A. Neusel \& A. Wetterer (Hrsg.), Vielfältige Verschiedenheiten: Geschlechterverhältnisse in Studium, Hochschule und Beruf (S. 193-220). Frankfurt a.M.: Campus.

Bandura, A. (1977). Self-efficacy: Toward a unifying theory of behavioral change. Psychological Review, 4, 191-215.

Becker-Schmidt, R. (2003). Zur doppelten Vergesellschaftung von Frauen. Gender-politik-online. URL: https://www.fu-berlin.de/sites/gpo/soz_eth/Geschlecht_als_Kategorie/Die_doppelte_Vergesellschaf tung_von_Frauen/index.html. Zugegriffen: Jan. 2021.

Bourdieu, P. (1976). Entwurf einer Theorie der Praxis auf der ethnologischen Grundlage der kabylischen Gesellschaft. Frankfurt a.M.: Suhrkamp.

Bourdieu, P. (1992). Die verborgenen Mechanismen der Macht. Hamburg: VSA.

Bourdieu, P. (1993). Sozialer Sinn: Kritik der theoretischen Vernunft. Frankfurt a.M.: Suhrkamp.

Bourdieu, P. (1997). Meditationen: Zur Kritik der scholastischen Vernunft. Frankfurt a.M.: Suhrkamp.

Bourdieu, P. (2014). Soziologische Fragen. 5. Aufl. Frankfurt a.M.: Suhrkamp.

Bourdieu, P., \& Wacquant, L. (1996). Reflexive Anthropologie. Frankfurt a.M.: Suhrkamp.

Bröckling, U. (2007). Das unternehmerische Selbst: Soziologie einer Subjektivierungsform. Frankfurt a.M.: Suhrkamp.

Clark, B. R. (1960). The „,cooling out“ function in higher education. The American Journal of Sociology, 65(6), 569-576.

Fischer, U. L. (2009). Anerkennung, Integration und Geschlecht: Zur Sinnstiftung des modernen Subjekts. Bielefeld: transcript.

Fürst, H. (2016). Handling rejection as failure: Aspiring writers getting the rejection slip. Valuation Studies, $4(2), 153-176$.

Gerhardt, U. (2001). Idealtypus: Zur methodischen Begründung der modernen Soziologie. Frankfurt a.M.: Suhrkamp.

Giegel, H.-J., Frank, G., \& Billerbeck, U. (1988). Industriearbeit und Selbstbehauptung: Berufsbiographische Orientierung und Gesundheitsverhalten in gefährdeten Lebensverhältnissen. Opladen: Leske + Budrich.

Glaser, B. G., Strauss, A. L., \& Paul, A. T. (2010). Grounded Theory: Strategien qualitativer Forschung. Bern: Huber.

Goffman, E. (1952). On cooling the mark out: Some aspects of adaptation to failure. Psychiatry, 15, 451-463.

Hänzi, D., \& Matthies, H. (2014). Leidenschaft - Pflicht - Not: Antriebsstrukturen und Erfolgskonzeptionen bei Spitzenkräften in Wissenschaft und Wirtschaft. In D. Hänzi, H. Matthies \& D. Simon (Hrsg.), Erfolg: Konstellationen und Paradoxien einer gesellschaftlichen Leitorientierung. Leviathan, Sonderband 29 (S. 246-264). Baden-Baden: Nomos.

Hardering, F. (2015). Meaningful work: Sinnvolle Arbeit zwischen Subjektivität, Arbeitsgestaltung und gesellschaftlichem Nutzen. Österreichische Zeitschrift für Soziologie, 40(4), 391-410.

Hardering, F. (2017). Wann erleben Beschäftigte ihre Arbeit als sinnvoll? Befunde aus einer Untersuchung über professionelle Dienstleistungsarbeit. Zeitschrift für Soziologie, 46(1), 39-54.

Hardering, F., \& Will-Zocholl, M. (2019). Zwischen Sinngestaltung und Sinnbewahrung - Aneignungsweisen hochqualifizierter Dienstleistungsarbeit. Berliner Journal für Soziologie, 29, 273-298.

Heinz, W. R. (1996). Soziale Benachteiligung und berufliche Förderung Jugendlicher im regionalen und internationalen Vergleich. Zeitschrift für Berufs- und Wirtschaftspädagogik, 92, 151-161.

Honneth, A. (2008). Arbeit und Anerkennung. Versuch einer Neubestimmung. Deutsche Zeitschrift für Philosophie, 56(3), 327-341.

Jansen, D. (2007). New forms of governance in research organizations: Disciplinary approaches, interfaces and integration. Dordrecht: Springer.

Jungbauer-Gans, M., \& Gross, C. (2013). Determinants of success in university careers: Findings from the German academic labor market. Zeitschrift für Soziologie, 42(1), 74-92.

Jurt, J. (2010). Die Habitus-Theorie von Pierre Bourdieu. Zeitschrift für Literatur- und Theatersoziologie, $3,5-17$.

Kahlert, H. (2011). „Cooling out“ und der riskante Weg an die Spitze: Zum Einfluss von Ungleichheitsregimes auf Karriereorientierungen im wissenschaftlichen Nachwuchs. In J. Wergen (Hrsg.), Von der Forschung zur Förderung: Promovierende im Blick der Hochschulen (S. 105-123). Münster: Lit. 
Kelle, U., \& Kluge, S. (1999). Vom Einzelfall zum Typus: Fallvergleich und Fallkontrastierung in der qualitativen Sozialforschung. Bd. 4. Wiesbaden: VS Verlag für Sozialwissenschaften.

Kleemann, F., \& Voß, G. G. (2010). Arbeit und Subjekt. In F. Böhle, G. G. Voss, G. Wachtler \& A. Hoffmann (Hrsg.), Handbuch Arbeitssoziologie (S. 415-450). Wiesbaden: VS Verlag für Sozialwissenschaften.

Krais, B. (2000). Das soziale Feld Wissenschaft und die Geschlechterverhältnisse. Theoretische Sondierungen. In B. Krais (Hrsg.), Wissenschaftskultur und Geschlechterordnung. Über die verborgenen Mechanismen männlicher Dominanz in der akademischen Welt (S. 31-54). Frankfurt a.M.: Campus.

Lenger, A., Schneickert, C., \& Schumacher, F. (2013). Pierre Bourdieus Konzeption des Habitus. In A. Lenger, C. Schneickert \& F. Schumacher (Hrsg.), Pierre Bourdieus Konzeption des Habitus: Grundlagen, Zugänge, Forschungsperspektiven (S. 11-41). Wiesbaden: Springer VS.

Lettke, F. (1996). Habitus und Strategien ostdeutscher Unternehmer. Eine qualitativ-empirische Analyse unternehmerischen Handelns. München: Rainer Hampp.

Lutter, M., \& Schröder, M. (2016). Who becomes a tenured professor, and why? Panel data evidence from German sociology, 1980-2013. Research Policy, 45(5), 999-1013.

Mainiero, L.A., \& Sullivan, S.E. (2005). Kaleidoscope careers: An alternate explanation for the „opt-out“ revolution. Academy of Management Executive, 19(1), 106-123.

Matthies, H. (2006). ,Entrepreneurshipping“ in unvollkommenen Märkten - das Beispiel der Wissenschaft. In K. Lohr \& H. M. Nickel (Hrsg.), Subjektivierung von Arbeit - Riskante Chancen (S. 149-179). Münster: Westfälisches Dampfboot.

Matthies, H., \& Rehbein, S. (2020). Inkongruenz. Ausstieg aus der Wissenschaft als Folge eines Mismatch zwischen Feld und Habitus. die hochschule, 29(1), 87-97.

Matthies, H., \& Torka, M. (2019). Academic habitus and institutional change: Comparing two generations of German scholars. Minerva, 57, 345-371.

Matthies, H., Simon, D., \& Torka, M. (Hrsg.). (2015). Die Responsivität der Wissenschaft: Wissenschaftliches Handeln in Zeiten neuer Wissenschaftspolitik. Bielefeld: transcript.

Mayntz, R. (1980). Gesetzgebung und Bürokratisierung. Wissenschaftliche Auswertung der Anhörung zu Ursachen einer Bürokratisierung in der öffentlichen Verwaltung, durchgeführt im Auftrag des Bundesministeriums des Innern. Bonn: BMI.

Miller, M. V., \& Robinson, C. (2004). Managing the disappointment of job termination: Outplacement as a cooling-out device. Journal of Applied Behavioral Science, 40(1), 49-65.

Mittelstrass, J. (1997). Der Flug der Eule. Von der Vernunft der Wissenschaft und der Aufgabe der Philosophie. Frankfurt a.M.: Suhrkamp.

Moldaschl, M. (Hrsg.). (2003). Subjektivierung von Arbeit. 2., überarb. und erw. Aufl. München: Hampp.

Nadai, E. (2007). Cooling out. Zur Aussortierung von „Leistungsschwachen“. In C. Arni, A. Glauser, C. Müller, M. Rychner, P. Schallberger \& C. Honegger (Hrsg.), Der Eigensinn des Materials. Erkundungen sozialer Wirklichkeit: Festschrift für Claudia Honegger zum 60. Geburtstag (S. 445-461). Frankfurt a.M.: Stroemfeld.

Nisic, N., \& Trübswetter, P. (2012). Berufswechsler in Deutschland und Großbritannien. IAB-Kurzbericht 1. URL: http://doku.iab.de/kurzber/2012/kb0112.pdf. Zugegriffen: Jan. 2021.

Oevermann, U. (1991). Genetischer Strukturalismus und das sozialwissenschaftliche Problem der Erklärung der Entstehung des Neuen. In S. Müller-Doohm (Hrsg.), Jenseits der Utopie: Theoriekritik der Gegenwart (S. 269-273). Frankfurt a.M.: Suhrkamp.

Oevermann, U. (1995). Ein Modell der Struktur von Religiosität. Zugleich ein Strukturmodell von Lebenspraxis und sozialer Zeit. In M. Wohlrab-Sahr (Hrsg.), Biographie und Religion. Zwischen Ritual und Selbstsuche (S. 27-102). Frankfurt a.M.: Campus.

Oevermann, U. (2009). Biographie, Krisenbewältigung und Bewährung. In S. Bartmann, A. Fehlhaber, S. Kirsch \& W. Lohfeld (Hrsg.), Natürlich stört das Leben ständig. Perspektiven auf Entwicklung und Erziehung (S. 35-55). Wiesbaden: VS Verlag für Sozialwissenschaften.

Penz, O., \& Sauer, B. (2016). Affektives Kapital: Die Ökonomisierung der Gefühle im Arbeitsleben. Frankfurt a.M.: Campus.

Rehbein, B., \& Saalman, G. (2014). Habitus (habitus). In G. Fröhlich \& B. Rehbein (Hrsg.), Bourdieu Handbuch: Leben - Werk - Wirkung (S. 110-118). Stuttgart: J.B. Metzler.

Schaeper, H., Kühn, T., \& Witzel, A. (2000). Diskontinuierliche Erwerbskarrieren und Berufswechsel in den 1990ern: Strukturmuster und biografische Umgangsweisen betrieblich ausgebildeter Fachkräfte. Mitteilungen aus der Arbeitsmarkt- und Berufsforschung, 33(1), 80-100.

Schelepa, S. (2010). Zur biographischen Deutung von Berufswechseln im Spannungsfeld von Autonomie und Heterenomie. In A. Bolder, R. Epping, R. Klein, G. Reuter \& A. Seiverth (Hrsg.), Neue Le- 
benslaufregimes - neue Konzepte der Bildung Erwachsener (S. 123-138). Wiesbaden: VS Verlag für Sozialwissenschaften.

Schmeiser, M. (2003). „Missratene“ Söhne und Töchter: Verlaufsformen des sozialen Abstiegs in Akademikerfamilien. Konstanz: UVK.

Seibert, N. (2007). Berufswechsel in Deutschland. Wenn der Schuster nicht bei seinem Leisten bleibt ... IAB-Kurzbericht Nr. 1. URL: http://hdl.handle.net/10419/158237. Zugegriffen: Jan. 2021.

Solga, H. (2004). Das Scheitern gering qualifizierter Jugendlicher an den Normalisierungspflichten moderner Bildungsgesellschaften. In M. Junge \& G. Lechner (Hrsg.), Scheitern: Aspekte eines sozialen Phänomens (S. 97-121). Wiesbaden: VS Verlag für Sozialwissenschaften.

Stauber, B., Pohl, A., \& Walther, A. (Hrsg.). (2007). Subjektorientierte Übergangsforschung: Rekonstruktion und Unterstützung biografischer Übergänge junger Erwachsener. Weinheim: Juventa.

Thomae, H. (1944). Das Wesen der menschlichen Antriebsstruktur. Leipzig: Ambrosius Barth.

Thomae, H. (1955). Persönlichkeit - eine dynamische Interpretation. 2., erw. Aufl. Bonn: H. Bouvier \& Co.

Thomas, G. M. (2014). Cooling the mother out: Revisiting and revising Goffman's account. Symbolic Interaction, 37(2), 283-299.

Wei, J. (2016). „I'm the next American idol“": Cooling out, accounts, and perseverance at reality talent show auditions. Symbolic Interaction, 39(1), 3-25.

Willems, H. (1997). Rahmen und Habitus. Zum theoretischen und methodischen Ansatz Erving Goffmans: Vergleiche, Anschlüsse und Anwendungen. Frankfurt a.M.: Suhrkamp.

Hildegard Matthies Dr. rer. pol., i.R., ehemals wissenschaftliche Mitarbeiterin am Wissenschaftszentrum Berlin für Sozialforschung. Forschungsschwerpunkte: Organisation, Profession, Biographie, Karriere und Geschlecht. Veröffentlichungen: (mit S. Rehbein) Inkongruenz: Ausstieg aus der Wissenschaft als Folge eines Mismatch zwischen Habitus und Feld, in: die hochschule. journal für wissenschaft und bildung, 2020; (mit M. Torka) Academic habitus and institutional change: Comparing two generations of German scholars, in: Minerva, 2019; Die Responsivität wissenschaftlicher Karrieren, in: H. Matthies, D. Simon \& M. Torka (Hrsg.), Responsivität der Wissenschaft. Wissenschaftliches Handeln in Zeiten neuer Wissenschaftspolitik, 2015. 